\title{
Article \\ Petrogenesis of Early Cretaceous High-Mg Adakitic Pluton in the North Lhasa Block, Tibet Plateau: Implications for the Tethyan Orogeny
}

\author{
Qian-Ru Xiao ${ }^{1} \mathbb{D}$, Yong-Feng Wei ${ }^{1,2, *}$, Zhong-Yuan Liu ${ }^{1}$, Fu-Hao Xiong ${ }^{1}$, Lei Fan ${ }^{1}$, Jian-Xin Wu ${ }^{1}$ and Han $\mathrm{Zhao}^{1}$ \\ 1 College of Earth Sciences, Chengdu University of Technology, Chengdu 610059, China; \\ xiaoqianru@cdut.edu.cn (Q.-R.X.); Liuzhongyuan43@hotmail.com (Z.-Y.L.); \\ xiongfuhao2014@cdut.edu.cn (F.-H.X.); fanl.vra@foxmail.com (L.F.); wujianxin34@hotmail.com (J.-X.W.); \\ zhaohan2013@cdut.edu.cn (H.Z.) \\ 2 Regional Geological Survey Team, Sichuan Geology and Mineral Bureau, Shuangliu 610213, China \\ * Correspondence: weiyongfeng1900@hotmail.com
}

check for

updates

Citation: Xiao, Q.-R.; Wei, Y.-F.;

Liu, Z.-Y.; Xiong, F.-H.; Fan, L.;

Wu, J.-X.; Zhao, H. Petrogenesis of

Early Cretaceous High-Mg Adakitic

Pluton in the North Lhasa Block,

Tibet Plateau: Implications for the

Tethyan Orogeny. Minerals 2022, 12,

213. https://doi.org/10.3390/

$\min 12020213$

Academic Editors: Sergei Khromykh and Andrei Tsygankov

Received: 12 December 2021

Accepted: 3 February 2022

Published: 7 February 2022

Publisher's Note: MDPI stays neutral with regard to jurisdictional claims in published maps and institutional affiliations.

Copyright: () 2022 by the authors Licensee MDPI, Basel, Switzerland. This article is an open access article distributed under the terms and conditions of the Creative Commons Attribution (CC BY) license (https:// creativecommons.org/licenses/by/ $4.0 /)$.

\begin{abstract}
Late Mesozoic igneous rocks from the north Lhasa block record the Neo-Tethyan orogeny in the southern Tibet Plateau. This study presents geochronological and geochemical data of Bieruozecuo quartz diorite pluton in the northern margin of the Lhasa block to constrain its petrogenesis and tectonic implications. The LA-ICP-MS zircon U-Pb geochronology of quartz diorites shows that the emplacement occurs at ca. 114-116 Ma, belonging to the products of Early Cretaceous magmatic activities. The high concentrations of $\mathrm{TiO}_{2}, \mathrm{MgO}$, and $\mathrm{MnO}$, together with $\mathrm{SiO}_{2}$ vs. $\mathrm{K}_{2} \mathrm{O}$ and $\mathrm{A} / \mathrm{NK}$ vs. A/CNK diagrams, all suggest that the Bieruozecuo quartz diorites are meta-aluminous and high-potassium calc-alkaline rocks. Their high $\mathrm{Sr}$ and low $\mathrm{Y}$ and $\mathrm{Yb}$ contents, as well as high $\mathrm{Sr} / \mathrm{Y}$ and $\mathrm{La} / \mathrm{Yb}$ ratios, are consistent with the typical adakitic rocks. The REE patterns show a large distribution of compositions, which have LREE, while HREE are buffered, along with large ratios of $(\mathrm{La} / \mathrm{Yb})_{\mathrm{N}}$, as well as high values of $\mathrm{K}_{2} \mathrm{O} / \mathrm{Na}_{2} \mathrm{O}, \mathrm{Mg} \#, \mathrm{Cr}$, and $\mathrm{Ni}$, all of which imply the partial melting of a delaminated lower crust, without obvious fractional crystallization during the magma ascending and emplacement. This study suggests that, with the closure of the Bangonghu-Nujiang Tethys Ocean Basin, the post-collisional extension of the north Lhasa block will have started no later than ca. 114-116 Ma. Combined with the previous studies, our new data demonstrates that the partial melting of the delaminated lower crust, in a post-collisional setting, may be the main mechanism responsible for the ca. 116-82 Ma adakitic magmatism in the north Lhasa block.
\end{abstract}

Keywords: Lhasa block; neo-tethyan orogeny; cretaceous; adakite; petrogenesis; tectonic setting

\section{Introduction}

The Lhasa block in the southern Tibet Plateau is characterized by the presence of large-scale Late Mesozoic-Cenozoic magmatic rocks, which records the magmatic-tectonic evolution and geodynamic processes during the oceanic lithosphere subduction and collision/accretion of terranes [1-6]. Hence, unraveling their petrogenesis is the key to understanding the tectonic evolution and Neo-Tethyan orogeny. Despite many studies that have been carried out on the Late Mesozoic magmatic rocks in the Lhasa block, the models, proposed so far on their petrogenesis and dynamic background, are still controversial. Some studies propose that the Late Mesozoic magmatic activity in the Lhasa block was formed during the northward subduction of the southern Yarlung Zangbo Neo-Tethyan -oceanic slab $[4,7,8]$. In contrast, others believe that their petrogenesis is closely related to the southward oceanic lithosphere subduction or terrane collision during the Northern Bangong Hu-Nujiang Tethys orogeny $[2,3,9,10]$. Meanwhile, previously published studies on the magma source of Late Mesozoic magmatic rocks in the Lhasa block are not consistent, suggesting different models, including remelting of the thickened lower crust in a 
collisional setting [7] and partial melting of Neo-Tethyan oceanic crust [2]. Therefore, it is necessary to continue to study the petrogenesis and tectonic setting of the Late Mesozoic magmatic rocks in the Lhasa block, to get a better understanding the Neo-Tethyan orogeny.

In this contribution, we present studies on the petrology, petrography, LA-ICP-MS zircon $\mathrm{U}-\mathrm{Pb}$ chronology, and whole-rock geochemistry of one Early Cretaceous quartz diorite pluton in the northern Lhasa block. Combined with published data of the other Late Mesozoic coeval magmatic rocks in northern and central Tibet, we discuss the melt sources and magmatic evolution processes, as well as their tectonic implications on the Neo-Tethyan orogeny in the Lhasa block, southern Tibet.

\section{Geological Setting and Petrography}

The Tibet Plateau, located in the eastern part of the Tethys tectonic domain, is a key area for studying the geodynamic processes of oceanic lithosphere subduction and collision of the Tethys terranes. Multiple Tethyan subductions and accretion orogenic processes occurred in Tibet Plateau, including the Early Paleozoic Proto-Tethys, Carboniferous-Triassic Paleo-Tethys, and Mesozoic-Cenozoic Neo-Tethys orogenies [11-15]. The Lhasa block is located in the southern Tibet Plateau, which is bound by the Yarlung Zangbo Neo-Tethyan Tethyan suture zone (IYZSZ) to the south and northern Bangonghu-Nujiang suture zone (BNSZ) to the north. Taking the Lobadui Milashan fault (LMF) and Shiquanhe-Namucuo ophiolite melange belt (SNMZ) as the boundaries, the Lhasa block is subdivided into the north Lhasa block (NL), central Lhasa block (CL), and south Lhasa block (SL) (Figure 1a). Mesozoic and Cenozoic magmatic rocks are the dominant rocks in the entire Lhasa block. Triassic-Cretaceous magmatic rocks, forming a large-scale Gandeses batholith belt, occur as the dominant rocks, only in the south Lhasa block, whereas the Late Jurassic/Early Cretaceous sedimentary strata, Zenong Group volcanic rocks, and Early Cretaceous intrusive rocks are widely exposed in the central and north Lhasa blocks $[8,16,17]$ (Figure 1b).

The Bieruozecuo quartz diorite pluton is located in the transitional part of the central and north Lhasa blocks. This area is characterized by the development of JurassicCretaceous volcanic sedimentary strata and a large number of intermediate-acid intrusive rocks (Figure 1b). The Early Cretaceous Bieruozecuo quartz diorite pluton is irregular or nearly oval in form, emplaced into the Early Cretaceous Shishenla and Duoni formations, with the acreage for a single mass rock of $10-25 \mathrm{~km}^{2}$. The investigated intrusion is about $19 \mathrm{~km}^{2}$. The petrographic observation of the outer rim of the pluton provides good evidence for the thermal contact metamorphism, including hornfelsification, silicification, and marbleization. The quartz diorite is a greyish-green color and displays a fine-grained texture. Plagioclase $(\sim 50 \%)$ is the most abundant mineral, followed by alkaline feldspar $(\sim 18 \%)$, quartz $(\sim 15 \%)$, hornblende $(\sim 9 \%)$, and biotite $(\sim 4 \%)$. The plagioclase group of minerals consists of oligoclase and andesine, in the form of subhedral crystals, with a diameter of $0.5-1.6 \mathrm{~mm}$. The euhedral growth zoning was generally observed in andesine. The main variety of alkaline feldspar is orthoclase. Most of the orthoclase grains are subhedral to anhedral. The quartz is heteromorphic. Alkali feldspar has xenomorphic granular structure. Zircon, apatite, magnetite, and sphene are rare, and other minerals are present only in trace amounts (Figure 2). The alterations cannot be observed in the fresh, thin sections of the samples. 

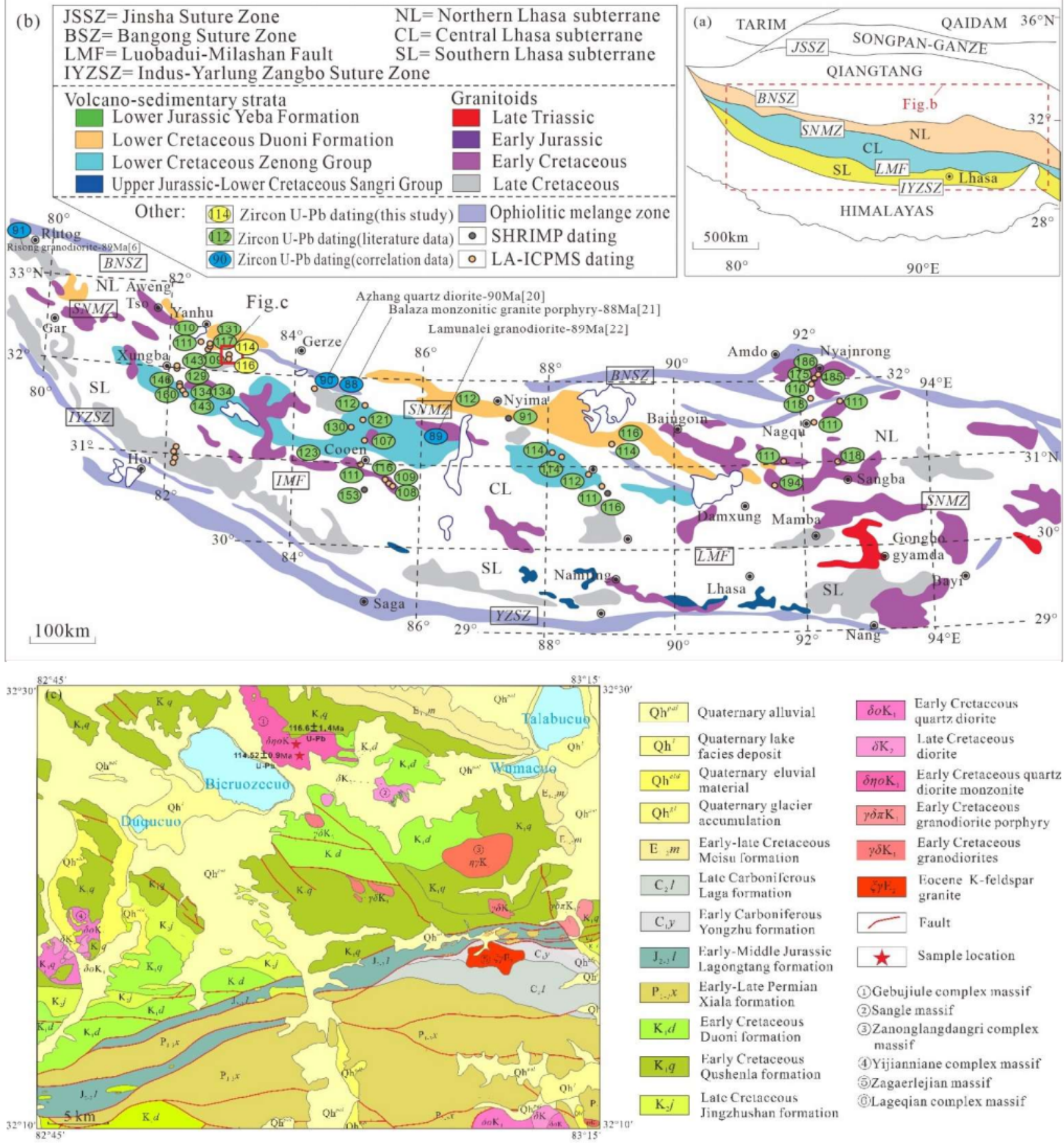

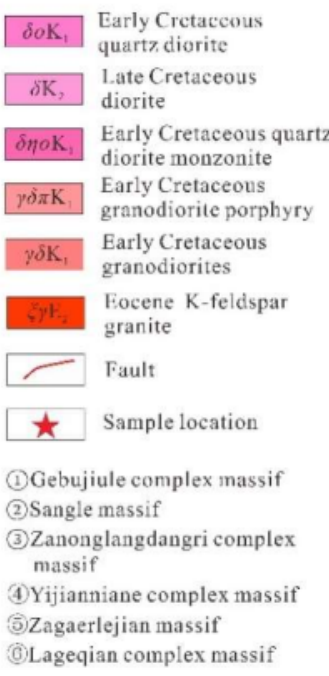

Figure 1. (a) Schematic tectonic framework of the Tibetan Plateau (modified from [18]); (b) tectonic framework of the Lhasa terrane, showing major tectonic subdivisions (modified from [19]; the correlation data are from [6,20-22]); (c) simplified geological map showing sample locations. 

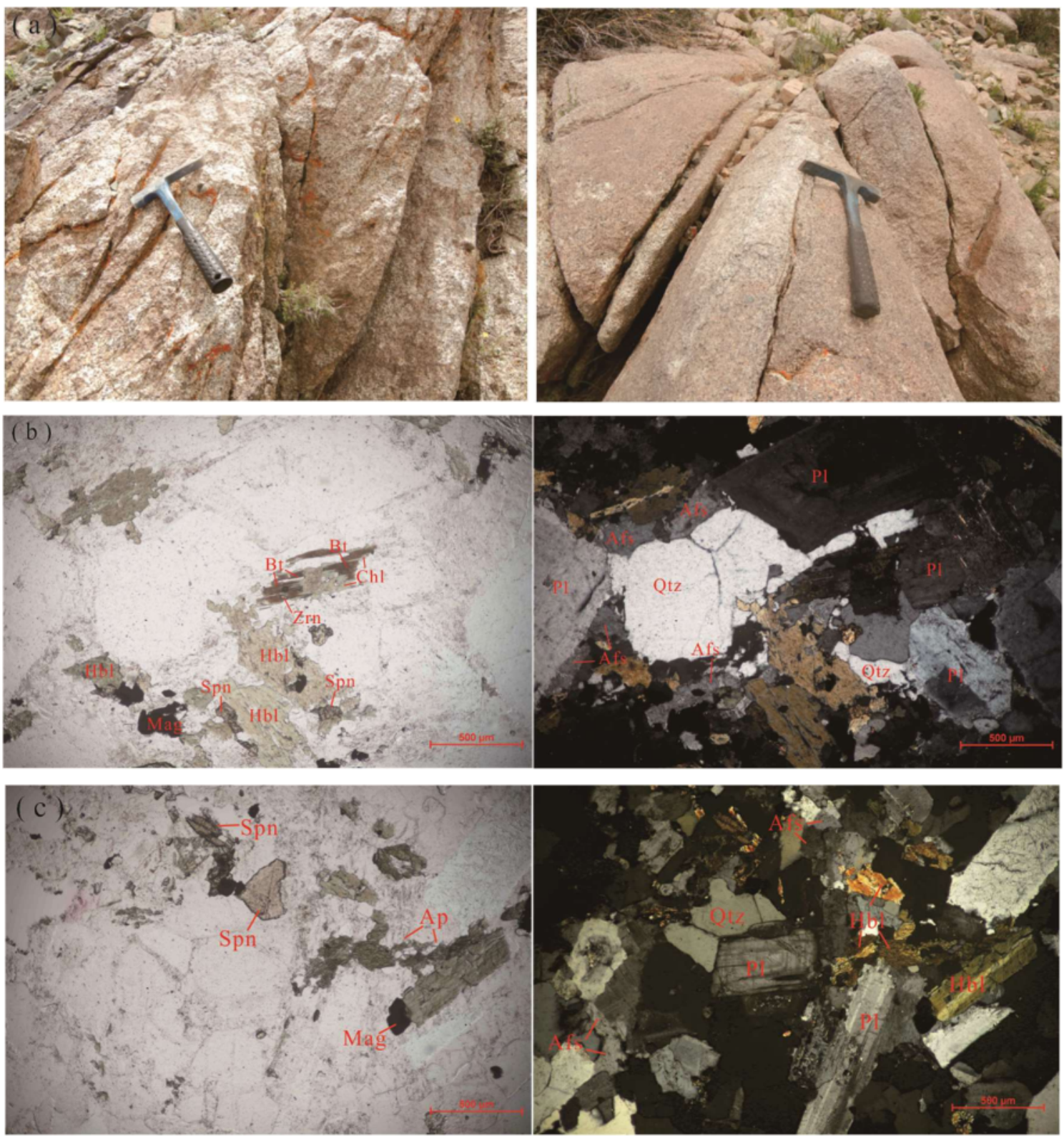

Figure 2. (a) Field photograph showing the outcrops of Bieruozecuo quartz diorite adakitic rocks; (b) Microphotograph of the adakitic rock (sample P10-19); (c) Microphotograph of the adakitic rock (sample P11-6). Qtz = quartz; Pl = plagioclase; Afs = alkali feldspar; Bt = biotite; $\mathrm{Hbl}=$ hornblende; Mag = magnetite; $\mathrm{Ap}=$ apatite; $\mathrm{Zrn}=$ zircon; $\mathrm{Chl}=$ chlorite; $\mathrm{Spn}=$ sphene.

\section{Samples and Methods}

Two samples of the Bieruozecuo quartz diorite were collected for zircon geochronology analysis, and sample P11(20) was collected from the inner of the pluton $\left(82^{\circ} 55^{\prime} 41^{\prime \prime} \mathrm{E}\right.$, $\left.32^{\circ} 20^{\prime} 04^{\prime \prime} \mathrm{N}\right)$, while sample P11(46) was collected from the rim of the pluton $\left(82^{\circ} 56^{\prime} 22^{\prime \prime} \mathrm{E}\right.$, $\left.32^{\circ} 19^{\prime} 53^{\prime \prime} \mathrm{N}\right)$. Prior to LA-ICP-MS analyses, the samples were pretreated, crushed, and sorted at the Huayang Rock and Mineral Testing Center of Sichuan, China. Zircon concentrates were mounted in epoxy mounts, which were then carefully polished. All zircon grains were observed under a microscope, with transmitted and reflected light, as well as with cathodoluminescence (CL) imaging to confirm high transparency and avoid measuring spots with inclusions or/and cracks. Measurements of $\mathrm{U}$ and $\mathrm{Pb}$ isotopes were carried out using LA-ICP-MS, equipped with an ArF 193 nm UV excimer laser, at the State Key Laboratory of Dynamics, Northwestern University, China. The single pulse energy was set 
to $220 \mathrm{~mJ}$, and the maximum laser repetition rate was $20 \mathrm{~Hz}$. A fluence of $50 \mathrm{Jcm}^{-2}$ was chosen, with a beam diameter of around $60 \mu \mathrm{m}$. The standard zircon 91500 was used as an external standard for the calibration of the $\mathrm{Pb}-\mathrm{U}$. For quality assurance, the standard was analyzed at the end of the analytical run, after the measurement of six samples. Further data evaluation has been performed by using ISOPLOT and SQUID software. More detailed analytical procedures are described by [23].

The bulk compositions (major, trace, and rare earth elements) of samples were performed using an Axios X-ray fluorescence spectrometer, X Series 2 plasma-mass spectrometer, IRIS Intripid2XSP ICP full spectrum direct-reading spectrometer, zeenit600 graphite furnace atomic absorption spectrometer, and AFS-230E atomic fluorescence spectrometer at Zhongnan Mineral Resources Supervision and Testing Center. The analytical uncertainties were less than $5 \%$.

\section{Results}

\subsection{U-Pb Zircon Age}

$\mathrm{U}-\mathrm{Pb}$ zircon data are shown in Table 1. Zircon grains from sample P11(20) and sample P11(46) are mostly euhedral to subhedral and 95 to $220 \mu \mathrm{m}$ in length. The zircons in the two samples show similar zircon texture; both the inherited cores and magmatic rims of the zircons were observed in the two studied samples. Most zircon grains are transparent and show euhedral concentric zoning under CL images (Figure 3). A total of 48 analyses of zircon grains were obtained (Table 1). The concordance curves of the quartz diorite in sample P11(20) are shown in Figure 4a. The concordant age within analytical errors is $114.36 .1 \pm 0.43 \mathrm{Ma}(\mathrm{MSWD}=0.44)$, yielding a weighted mean ${ }^{206} \mathrm{~Pb} /{ }^{238} \mathrm{U}$ age of $114.52 \pm 0.91 \mathrm{Ma}$ (mean squared weighted deviation $(\mathrm{MSWD})=0.87$ ). The concordance age of zircons in sample P11(46) is shown in Figure $4 b$, and its concordance age is $116.7 \pm 0.70 \mathrm{Ma}(\mathrm{MSWD}=7.7)$, with a weighted mean ${ }^{206} \mathrm{~Pb} /{ }^{238} \mathrm{U}$ age of $116.6 \pm 1.4 \mathrm{Ma}$ $(\mathrm{MSWD}=0.95)$. Therefore, the crystallization age of the Bieruozecuo quartz diorite is $116 \sim 114 \mathrm{Ma}$ (Early Cretaceous).

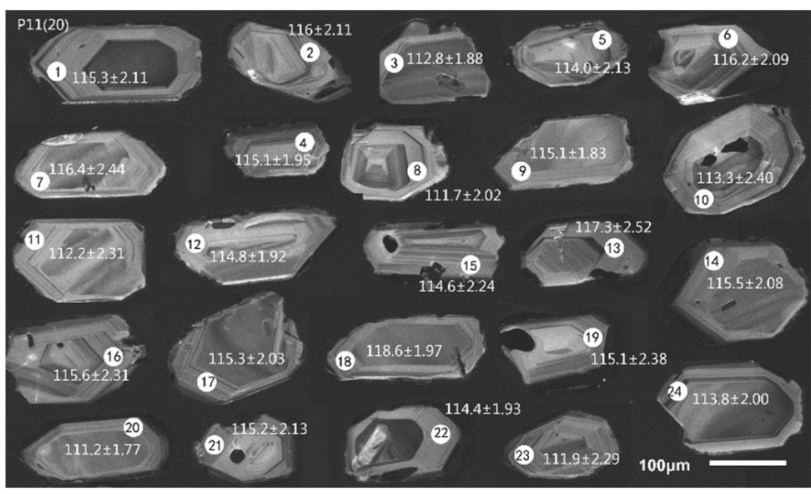

(a)

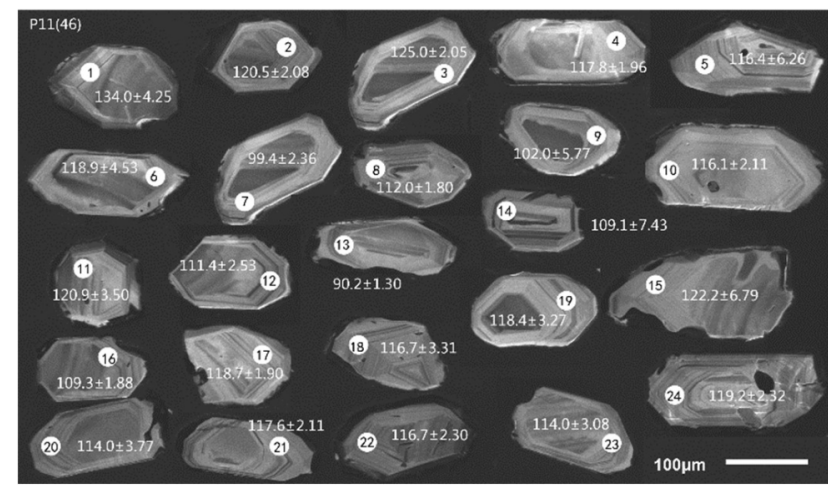

(b)

Figure 3. Representative cathodoluminescence images of zircon grains, analyzed from (a) sample 11(20) and (b) sample 11(46). 
Table 1. LA-ICP-MS zircon U-Pb ages for samples from the Bieruozecuo pluton in the Lhasa block, southern Tibet.

\begin{tabular}{|c|c|c|c|c|c|c|c|c|c|c|c|c|c|c|c|c|}
\hline \multirow{3}{*}{$\begin{array}{c}\text { Sample } \\
\text { Mark }\end{array}$} & \multicolumn{8}{|c|}{ Isotope Ratio } & \multicolumn{8}{|c|}{ Age (Ma) } \\
\hline & \multicolumn{2}{|c|}{${ }^{207} \mathrm{~Pb} /{ }^{206} \mathrm{~Pb}$} & \multicolumn{2}{|c|}{${ }^{207} \mathrm{~Pb} /{ }^{235} \mathrm{U}$} & \multicolumn{2}{|c|}{${ }^{206} \mathrm{~Pb} /{ }^{238} \mathrm{U}$} & \multicolumn{2}{|c|}{${ }^{208} \mathrm{~Pb} /{ }^{232} \mathrm{Th}$} & \multicolumn{2}{|c|}{${ }^{207} \mathrm{~Pb} /{ }^{206} \mathrm{~Pb}$} & \multicolumn{2}{|c|}{${ }^{207} \mathrm{~Pb} /{ }^{235} \mathrm{U}$} & \multicolumn{2}{|c|}{${ }^{206} \mathrm{~Pb} /{ }^{238} \mathrm{U}$} & \multicolumn{2}{|c|}{${ }^{208} \mathrm{~Pb} /{ }^{232} \mathrm{Th}$} \\
\hline & Ratio & $1 \sigma$ & Ratio & $1 \sigma$ & Ratio & $1 \sigma$ & Ratio & $1 \sigma$ & Age & $1 \sigma$ & Age & $1 \sigma$ & Age & $1 \sigma$ & Age & $1 \sigma$ \\
\hline P11(20)-01 & 0.04709 & 0.00366 & 0.11723 & 0.00838 & 0.01805 & 0.00033 & 0.00558 & 0.00021 & 53.3 & 176.17 & 112.6 & 7.62 & 115.3 & 2.11 & 112.4 & 4.28 \\
\hline P11(20)-02 & 0.05460 & 0.00384 & 0.13679 & 0.00867 & 0.01816 & 0.00033 & 0.00613 & 0.00025 & 395.9 & 150.5 & 130.2 & 7.75 & 116 & 2.11 & 123.5 & 4.95 \\
\hline P11(20)-03 & 0.04615 & 0.00295 & 0.11235 & 0.00634 & 0.01765 & 0.00030 & 0.00562 & 0.00017 & 5.7 & 146.94 & 108.1 & 5.79 & 112.8 & 1.88 & 113.3 & 3.48 \\
\hline P11(20)-04 & 0.05072 & 0.00313 & 0.12603 & 0.00682 & 0.01802 & 0.00031 & 0.00562 & 0.00018 & 228.3 & 136.7 & 120.5 & 6.15 & 115.1 & 1.95 & 113.3 & 3.66 \\
\hline P11(20)-05 & 0.05027 & 0.00395 & 0.12374 & 0.00895 & 0.01785 & 0.00034 & 0.00572 & 0.00023 & 207.3 & 172.58 & 118.5 & 8.09 & 114 & 2.13 & 115.3 & 4.53 \\
\hline P11(20)-07 & 0.04935 & 0.00474 & 0.12399 & 0.01123 & 0.01822 & 0.00039 & 0.00614 & 0.00028 & 164.5 & 210.23 & 118.7 & 10.15 & 116.4 & 2.44 & 123.7 & 5.67 \\
\hline P11(20)-08 & 0.04721 & 0.00348 & 0.11380 & 0.00764 & 0.01748 & 0.00032 & 0.00584 & 0.00019 & 59.5 & 167.44 & 109.4 & 6.97 & 111.7 & 2.02 & 117.7 & 3.88 \\
\hline P11(20)-09 & 0.04539 & 0.00265 & 0.11280 & 0.00568 & 0.01802 & 0.00029 & 0.00579 & 0.00014 & 0.1 & 101.05 & 108.5 & 5.18 & 115.1 & 1.83 & 116.7 & 2.84 \\
\hline P11(20)-10 & 0.04063 & 0.00497 & 0.09935 & 0.01172 & 0.01773 & 0.00038 & 0.00553 & 0.00028 & 0.1 & 0 & 96.2 & 10.83 & 113.3 & 2.4 & 111.5 & 5.68 \\
\hline P11(20)-11 & 0.04552 & 0.00449 & 0.11021 & 0.01028 & 0.01756 & 0.00036 & 0.00560 & 0.00025 & 0.1 & 194.99 & 106.2 & 9.4 & 112.2 & 2.31 & 112.9 & 4.95 \\
\hline P11(20)-12 & 0.05094 & 0.00309 & 0.12626 & 0.00667 & 0.01797 & 0.00030 & 0.00600 & 0.00016 & 237.9 & 134.15 & 120.7 & 6.02 & 114.8 & 1.92 & 121 & 3.16 \\
\hline P11(20)-13 & 0.05285 & 0.00532 & 0.13382 & 0.01275 & 0.01836 & 0.00040 & 0.00624 & 0.00031 & 322.4 & 213.24 & 127.5 & 11.42 & 117.3 & 2.52 & 125.7 & 6.26 \\
\hline P11(20)-14 & 0.05341 & 0.00364 & 0.13311 & 0.00810 & 0.01807 & 0.00033 & 0.00616 & 0.00020 & 346.1 & 146.56 & 126.9 & 7.26 & 115.5 & 2.08 & 124.2 & 3.93 \\
\hline P11(20)-15 & 0.05874 & 0.00454 & 0.14532 & 0.01028 & 0.01794 & 0.00035 & 0.00672 & 0.00026 & 557.4 & 160.27 & 137.8 & 9.11 & 114.6 & 2.24 & 135.4 & 5.28 \\
\hline P11(20)-18 & 0.04951 & 0.00306 & 0.12683 & 0.00686 & 0.01857 & 0.00031 & 0.00611 & 0.00017 & 172.2 & 138.15 & 121.2 & 6.18 & 118.6 & 1.97 & 123 & 3.5 \\
\hline P11(20)-19 & 0.05166 & 0.00469 & 0.12832 & 0.01089 & 0.01801 & 0.00038 & 0.00602 & 0.00026 & 270.5 & 194.89 & 122.6 & 9.8 & 115.1 & 2.38 & 121.3 & 5.24 \\
\hline P11(20)-20 & 0.04922 & 0.00284 & 0.11816 & 0.00586 & 0.01741 & 0.00028 & 0.00584 & 0.00014 & 158.5 & 129.68 & 113.4 & 5.32 & 111.2 & 1.77 & 117.6 & 2.82 \\
\hline P11(20)-21 & 0.06305 & 0.00417 & 0.15674 & 0.00917 & 0.01803 & 0.00034 & 0.00621 & 0.00023 & 709.8 & 134.61 & 147.8 & 8.05 & 115.2 & 2.13 & 125.1 & 4.61 \\
\hline P11(20)-22 & 0.04764 & 0.00313 & 0.11760 & 0.00688 & 0.01790 & 0.00031 & 0.00594 & 0.00019 & 80.8 & 149.81 & 112.9 & 6.25 & 114.4 & 1.93 & 119.7 & 3.73 \\
\hline P11(20)-23 & 0.04929 & 0.00450 & 0.11904 & 0.01018 & 0.01751 & 0.00036 & 0.00584 & 0.00025 & 161.7 & 200.42 & 114.2 & 9.24 & 111.9 & 2.29 & 117.6 & 4.99 \\
\hline P11(20)-24 & 0.05000 & 0.00352 & 0.12278 & 0.00781 & 0.01780 & 0.00032 & 0.00595 & 0.00021 & 195.2 & 155.96 & 117.6 & 7.06 & 113.8 & 2 & 119.9 & 4.16 \\
\hline P11(46)-01 & 0.05093 & 0.00732 & 0.14750 & 0.02037 & 0.02100 & 0.00067 & 0.00745 & 0.00052 & 237.5 & 300.97 & 139.7 & 18.02 & 134 & 4.25 & 149.9 & 10.35 \\
\hline P11(46)-02 & 0.05078 & 0.00363 & 0.13211 & 0.00844 & 0.01887 & 0.00033 & 0.00660 & 0.00021 & 230.9 & 157.24 & 126 & 7.57 & 120.5 & 2.08 & 132.9 & 4.21 \\
\hline P11(46)-03 & 0.05130 & 0.00358 & 0.13846 & 0.00861 & 0.01957 & 0.00032 & 0.00673 & 0.00022 & 254.2 & 152.94 & 131.7 & 7.68 & 125 & 2.05 & 135.5 & 4.49 \\
\hline P11(46)-04 & 0.05182 & 0.00352 & 0.13182 & 0.00793 & 0.01845 & 0.00031 & 0.00649 & 0.00021 & 277.4 & 148.45 & 125.7 & 7.11 & 117.8 & 1.96 & 130.8 & 4.18 \\
\hline P11(46)-05 & 0.04936 & 0.01251 & 0.12403 & 0.03065 & 0.01822 & 0.00099 & 0.00850 & 0.00090 & 164.7 & 504.78 & 118.7 & 27.69 & 116.4 & 6.26 & 171 & 17.94 \\
\hline
\end{tabular}


Table 1. Cont.

\begin{tabular}{|c|c|c|c|c|c|c|c|c|c|c|c|c|c|c|c|c|}
\hline \multirow{3}{*}{$\begin{array}{c}\text { Sample } \\
\text { Mark }\end{array}$} & \multicolumn{8}{|c|}{ Isotope Ratio } & \multicolumn{8}{|c|}{ Age (Ma) } \\
\hline & \multicolumn{2}{|c|}{${ }^{207} \mathrm{~Pb} /{ }^{206} \mathrm{~Pb}$} & \multicolumn{2}{|c|}{${ }^{207} \mathrm{~Pb} /{ }^{235} \mathrm{U}$} & \multicolumn{2}{|c|}{${ }^{206} \mathrm{~Pb} /{ }^{238} \mathrm{U}$} & \multicolumn{2}{|c|}{${ }^{208} \mathrm{~Pb} / 232 \mathrm{Th}$} & \multicolumn{2}{|c|}{${ }^{207} \mathrm{~Pb} /{ }^{206} \mathrm{~Pb}$} & \multicolumn{2}{|c|}{${ }^{207} \mathrm{~Pb} /{ }^{235} \mathrm{U}$} & \multicolumn{2}{|c|}{${ }^{206} \mathrm{~Pb} /{ }^{238} \mathrm{U}$} & \multicolumn{2}{|c|}{${ }^{208} \mathrm{~Pb} /{ }^{232} \mathrm{Th}$} \\
\hline & Ratio & $1 \sigma$ & Ratio & $1 \sigma$ & Ratio & $1 \sigma$ & Ratio & $1 \sigma$ & Age & $1 \sigma$ & Age & $1 \sigma$ & Age & $1 \sigma$ & Age & $1 \sigma$ \\
\hline P11(46)-07 & 0.04786 & 0.00684 & 0.10252 & 0.01419 & 0.01553 & 0.00037 & 0.00529 & 0.00033 & 91.2 & 308.93 & 99.1 & 13.07 & 99.4 & 2.36 & 106.7 & 6.7 \\
\hline P11(46)-08 & 0.04922 & 0.00339 & 0.11891 & 0.00728 & 0.01752 & 0.00028 & 0.00574 & 0.00019 & 158.2 & 153.49 & 114.1 & 6.6 & 112 & 1.8 & 115.6 & 3.78 \\
\hline P11(46)-09 & 0.04935 & 0.01375 & 0.10852 & 0.02954 & 0.01594 & 0.00091 & 0.00596 & 0.00062 & 164.4 & 547.07 & 104.6 & 27.06 & 102 & 5.77 & 120.1 & 12.4 \\
\hline P11(46)-10 & 0.05110 & 0.00404 & 0.12808 & 0.00925 & 0.01818 & 0.00033 & 0.00627 & 0.00024 & 245.2 & 172.54 & 122.4 & 8.33 & 116.1 & 2.11 & 126.2 & 4.86 \\
\hline P11(46)-12 & 0.05022 & 0.00528 & 0.12070 & 0.01198 & 0.01743 & 0.00040 & 0.00635 & 0.00031 & 205 & 226.96 & 115.7 & 10.86 & 111.4 & 2.53 & 128 & 6.28 \\
\hline P11(46)-13 & 0.14693 & 0.00607 & 0.28555 & 0.00794 & 0.01409 & 0.00020 & 0.00960 & 0.00017 & 2310.5 & 69.22 & 255.1 & 6.27 & 90.2 & 1.3 & 193.1 & 3.47 \\
\hline P11(46)-14 & 0.05317 & 0.01613 & 0.12522 & 0.03702 & 0.01708 & 0.00117 & 0.00653 & 0.00106 & 336 & 571.83 & 119.8 & 33.41 & 109.1 & 7.43 & 131.5 & 21.25 \\
\hline P11(46)-15 & 0.05118 & 0.01302 & 0.13507 & 0.03348 & 0.01914 & 0.00107 & 0.00930 & 0.00109 & 248.8 & 499.44 & 128.6 & 29.95 & 122.2 & 6.79 & 187.1 & 21.76 \\
\hline P11(46)-16 & 0.05242 & 0.00385 & 0.12360 & 0.00819 & 0.01710 & 0.00030 & 0.00550 & 0.00016 & 303.7 & 158.38 & 118.3 & 7.4 & 109.3 & 1.88 & 110.9 & 3.27 \\
\hline P11(46)-17 & 0.05034 & 0.00342 & 0.12904 & 0.00780 & 0.01859 & 0.00030 & 0.00682 & 0.00021 & 210.9 & 150.2 & 123.2 & 7.02 & 118.7 & 1.9 & 137.4 & 4.25 \\
\hline P11(46)-18 & 0.05145 & 0.00644 & 0.12966 & 0.01546 & 0.01827 & 0.00052 & 0.00733 & 0.00044 & 261.1 & 264.1 & 123.8 & 13.9 & 116.7 & 3.31 & 147.7 & 8.92 \\
\hline P11(46)-19 & 0.05268 & 0.00621 & 0.13472 & 0.01508 & 0.01854 & 0.00052 & 0.00612 & 0.00040 & 315 & 248.02 & 128.3 & 13.49 & 118.4 & 3.27 & 123.4 & 7.95 \\
\hline P11(46)-20 & 0.05305 & 0.00772 & 0.13047 & 0.01824 & 0.01783 & 0.00060 & 0.00722 & 0.00052 & 330.8 & 300.38 & 124.5 & 16.38 & 114 & 3.77 & 145.4 & 10.49 \\
\hline P11(46)-22 & 0.05202 & 0.00425 & 0.13105 & 0.00982 & 0.01827 & 0.00036 & 0.00597 & 0.00021 & 286.3 & 176.06 & 125 & 8.82 & 116.7 & 2.3 & 120.3 & 4.19 \\
\hline P11(46)-23 & 0.05078 & 0.00602 & 0.12497 & 0.01408 & 0.01785 & 0.00049 & 0.00576 & 0.00030 & 230.7 & 252.21 & 119.6 & 12.71 & 114 & 3.08 & 116.1 & 6.1 \\
\hline P11(46)-24 & 0.05174 & 0.00463 & 0.13314 & 0.01110 & 0.01866 & 0.00037 & 0.00627 & 0.00024 & 274.1 & 192.25 & 126.9 & 9.95 & 119.2 & 2.32 & 126.4 & 4.89 \\
\hline
\end{tabular}




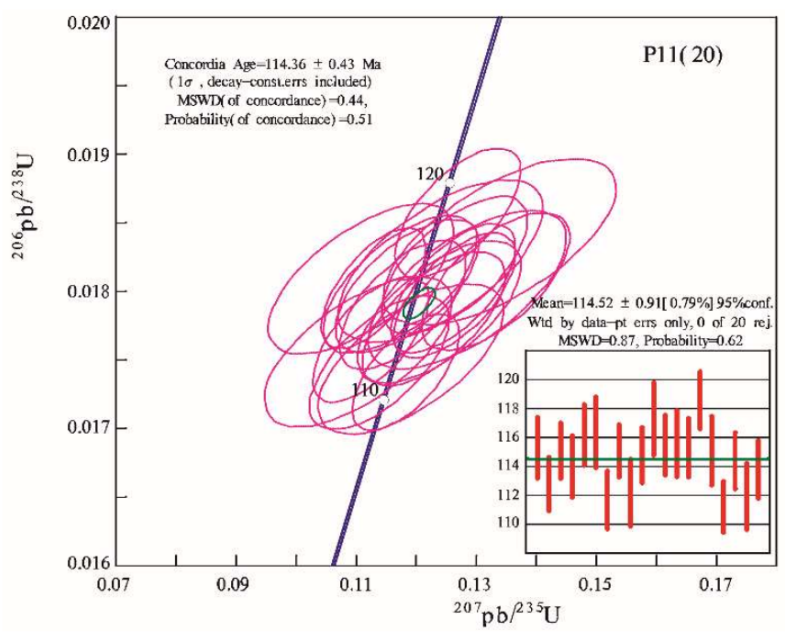

(a)

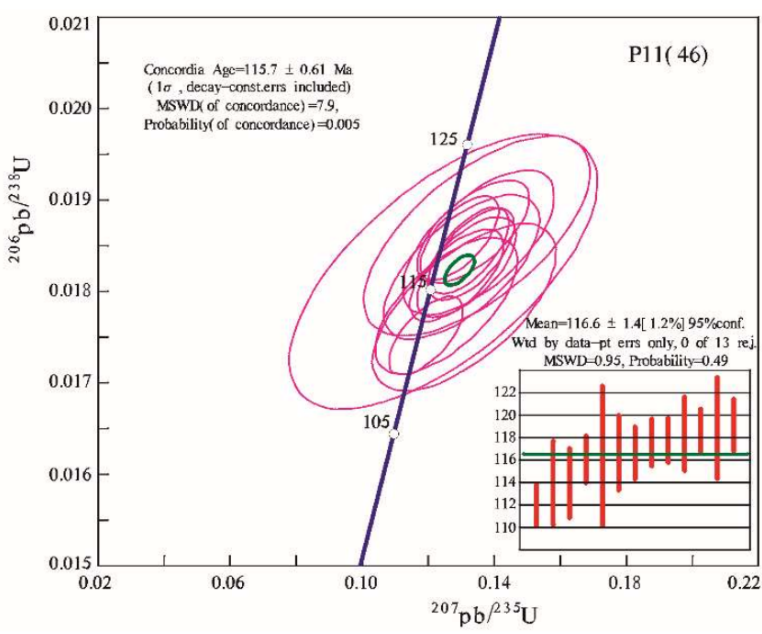

(b)

Figure 4. Concordia diagram for zircons from the samples; the circles indicate the locations of LA-ICP-MS U-Pb dating spot analyses. (a) P11(20); (b) P11(46).

\subsection{Bulk Geochemical Characteristics}

The major and trace element compositions of the representative samples $(\mathrm{N}=15)$ from the Bieruozecuo pluton are presented in Table 2. The studied samples have low contents of LOI, indicating that the samples are fresh, and the geochemical data can be used to efficiently constain their petregenesis. They contain higher $\mathrm{Al}_{2} \mathrm{O}_{3}(14.72-15.75 \mathrm{wt} . \%)$, and $\mathrm{Na}_{2} \mathrm{O}$ (3.27-4.02 wt.\%), but the lower concentrations of $\mathrm{MgO}(1.60-2.67 \mathrm{wt} . \%)$ and $\mathrm{TiO}_{2}$ (0.45-0.71 wt.\%). ALK, $\mathrm{Na}_{2} \mathrm{O} / \mathrm{K}_{2} \mathrm{O}$ ratios, and $\mathrm{A} / \mathrm{CNK}$ ratios are 6.50-7.26\%, 0.95-1.49, $0.89-0.93$, respectively. The studied rocks show high-K calc-alkaline affinity (Figure 5a) and fall into the meta-aluminous field in the A/NK-A/CNK diagram (Figure 5b). The Bieruozecuo quartz diorites show a narrow range in $\mathrm{SiO}_{2}$, from 64.07 to $68.32 \mathrm{wt} . \%$, and all samples are plotted in the granodiorite field on the total alkalis-silica diagram (Figure 6a). The Th vs. Co diagram suggests that all samples are depleted in Co and Th and plot in the calc-alkaline field (Figure $6 \mathrm{~b}$ ).
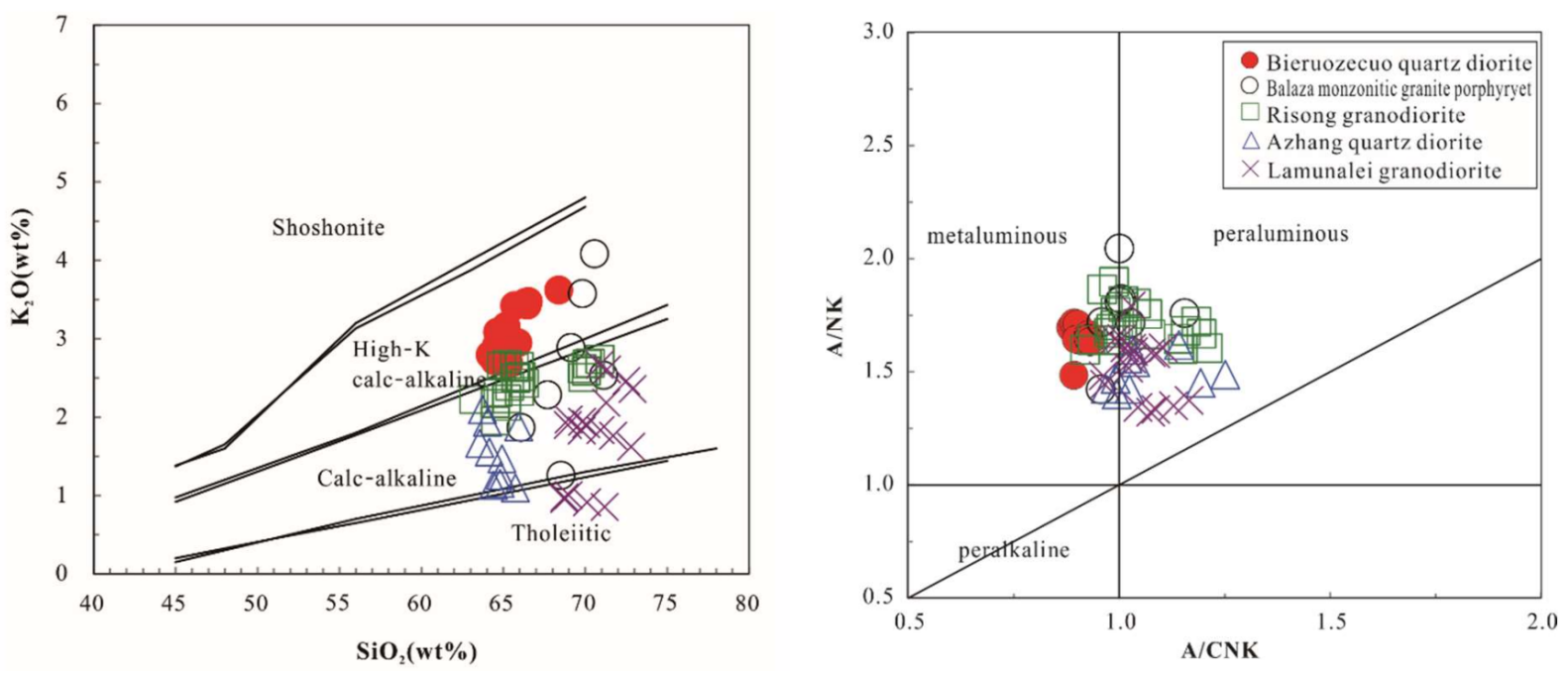

Figure 5. Diagram showing the variations in $\mathrm{SiO}_{2}$ and $\mathrm{K}_{2} \mathrm{O}$ concentrations of the Bieruozecuo quartz diorites and other Late Cretaceous igneous rocks within the central and northern Lhasa blocks, as well as the Miocene adakitic rocks in the southern Lhasa block. Data sources: ore-bearing porphyry in Balazha, [21]; adakitic rocks in Rutog, [24]; adakitic rocks in Azhang, [20]; adakitic rocks in Lamunale, [22]. 
Table 2. Major elements (wt\%), trace elements (ppm) and REE (ppm) analyses of the Bieruozecuo quartz diorites.

\begin{tabular}{|c|c|c|c|c|c|c|c|c|c|c|c|c|c|c|c|}
\hline Samples & P10(19) & P10(88) & P10(130) & P10(161) & P10(xx1) & $P 10(x \times 2)$ & P10(xx3) & P10(xx4) & P10(xx5) & P11(6) & P11(8) & P11(18) & P11(20) & P11(34) & P11(41) \\
\hline Lithology & \multicolumn{15}{|c|}{ Quartz Diorite } \\
\hline $\mathrm{SiO}_{2}$ & 65.640 & 64.840 & 64.500 & 68.320 & 64.430 & 64.910 & 64.070 & 64.280 & 65.730 & 65.170 & 66.410 & 65.650 & 65.980 & 66.480 & 66.280 \\
\hline $\mathrm{Al}_{2} \mathrm{O}_{3}$ & 15.690 & 15.440 & 15.440 & 14.720 & 15.320 & 15.470 & 15.710 & 15.750 & 15.460 & 15.410 & 15.090 & 14.940 & 15.340 & 15.040 & 14.920 \\
\hline $\mathrm{Fe}_{2} \mathrm{O}_{3}$ & 1.790 & 1.860 & 1.890 & 1.260 & 2.110 & 1.980 & 2.050 & 2.100 & 1.820 & 1.410 & 1.140 & 1.380 & 1.170 & 1.830 & 1.290 \\
\hline $\mathrm{FeO}$ & 1.940 & 2.280 & 2.080 & 1.590 & 2.330 & 2.300 & 2.270 & 2.150 & 1.950 & 2.970 & 2.890 & 2.830 & 2.610 & 2.330 & 2.800 \\
\hline $\mathrm{CaO}$ & 4.130 & 4.340 & 4.210 & 3.620 & 4.320 & 4.120 & 4.660 & 4.660 & 4.060 & 4.460 & 3.890 & 4.150 & 4.030 & 3.900 & 3.790 \\
\hline $\mathrm{MgO}$ & 2.140 & 2.530 & 2.400 & 1.600 & 2.670 & 2.440 & 2.540 & 2.430 & 2.140 & 2.060 & 1.840 & 1.990 & 1.760 & 1.830 & 1.830 \\
\hline $\mathrm{K}_{2} \mathrm{O}$ & 2.700 & 2.830 & 3.080 & 3.620 & 2.900 & 3.040 & 2.790 & 2.710 & 2.940 & 3.160 & 3.470 & 3.420 & 3.430 & 3.430 & 3.460 \\
\hline $\mathrm{TiO}_{2}$ & 0.565 & 0.662 & 0.617 & 0.450 & 0.713 & 0.690 & 0.654 & 0.630 & 0.580 & 0.587 & 0.560 & 0.595 & 0.511 & 0.569 & 0.552 \\
\hline $\mathrm{P}_{2} \mathrm{O}_{5}$ & 0.189 & 0.235 & 0.228 & 0.160 & 0.253 & 0.239 & 0.264 & 0.266 & 0.238 & 0.115 & 0.112 & 0.129 & 0.102 & 0.115 & 0.123 \\
\hline $\mathrm{MnO}$ & 0.064 & 0.063 & 0.069 & 0.058 & 0.073 & 0.066 & 0.067 & 0.062 & 0.059 & 0.075 & 0.073 & 0.072 & 0.073 & 0.073 & 0.078 \\
\hline LOI & 0.767 & 0.787 & 1.440 & 0.653 & 0.777 & 0.603 & 0.697 & 0.747 & 0.832 & 0.712 & 0.765 & 1.040 & 1.220 & 0.713 & 1.160 \\
\hline Total & 100.462 & 99.714 & 99.758 & 99.896 & 99.788 & 99.826 & 99.802 & 99.678 & 99.740 & 99.982 & 99.823 & 99.939 & 99.635 & 100.016 & 99.873 \\
\hline Mg\# & 51.792 & 53.286 & 53.087 & 51.151 & 52.953 & 51.588 & 52.390 & 51.744 & 51.533 & 46.418 & 45.581 & 46.558 & 46.136 & 45.064 & 45.163 \\
\hline$\sigma$ & 1.990 & 1.930 & 2.090 & 2.070 & 1.990 & 2.050 & 2.030 & 1.960 & 1.940 & 1.920 & 1.930 & 1.950 & 1.970 & 1.910 & 1.920 \\
\hline $\mathrm{A} / \mathrm{CNK}$ & 0.921 & 0.906 & 0.909 & 0.893 & 0.900 & 0.917 & 0.886 & 0.893 & 0.926 & 0.901 & 0.930 & 0.899 & 0.928 & 0.927 & 0.932 \\
\hline A/NK & 1.645 & 1.687 & 1.653 & 1.486 & 1.669 & 1.650 & 1.697 & 1.718 & 1.659 & 1.713 & 1.649 & 1.645 & 1.666 & 1.648 & 1.635 \\
\hline $\mathrm{Cu}$ & 9.320 & 22.000 & 27.000 & 6.360 & 18.800 & 44.500 & 19.600 & 25.600 & 29.400 & 33.400 & 29.800 & 22.200 & 24.900 & 29.000 & 29.900 \\
\hline $\mathrm{Pb}$ & 9.280 & 18.800 & 14.500 & 13.600 & 22.300 & 29.300 & 22.100 & 19.500 & 22.000 & 13.900 & 15.300 & 15.800 & 7.040 & 14.200 & 20.700 \\
\hline $\mathrm{Zn}$ & 53.000 & 67.700 & 61.700 & 46.000 & 71.800 & 71.600 & 71.400 & 61.200 & 63.500 & 69.200 & 53.600 & 49.600 & 49.200 & 50.700 & 69.200 \\
\hline $\mathrm{Cr}$ & 44.400 & 63.500 & 50.200 & 32.500 & 59.200 & 50.900 & 57.500 & 56.300 & 48.100 & 32.800 & 20.800 & 27.000 & 23.800 & 22.800 & 23.100 \\
\hline $\mathrm{Ni}$ & 22.400 & 35.500 & 28.200 & 20.400 & 32.000 & 29.500 & 30.300 & 28.400 & 25.400 & 13.500 & 10.800 & 12.200 & 10.400 & 11.000 & 11.300 \\
\hline Co & 13.700 & 19.200 & 17.200 & 9.270 & 19.100 & 17.300 & 16.600 & 17.000 & 16.500 & 18.400 & 17.000 & 16.600 & 12.500 & 15.500 & 16.200 \\
\hline $\mathrm{Rb}$ & 65.000 & 107.000 & 85.200 & 111.000 & 114.000 & 131.000 & 98.900 & 95.300 & 112.000 & 125.000 & 129.000 & 132.000 & 124.000 & 129.000 & 139.000 \\
\hline Cs & 5.760 & 4.710 & 6.500 & 6.100 & 6.670 & 8.980 & 5.330 & 5.350 & 5.530 & 3.340 & 4.110 & 5.540 & 4.000 & 4.100 & 4.620 \\
\hline $\mathrm{W}$ & 47.500 & 82.600 & 74.200 & 27.700 & 79.200 & 60.200 & 58.100 & 70.800 & 77.000 & 76.200 & 83.300 & 65.400 & 30.800 & 60.400 & 71.200 \\
\hline $\mathrm{Sb}$ & 0.270 & 0.450 & 0.260 & 0.220 & 0.660 & 1.240 & 0.410 & 0.850 & 0.540 & 1.240 & 1.180 & 0.440 & 0.200 & 0.560 & 2.070 \\
\hline $\mathrm{Bi}$ & 0.081 & 0.120 & 0.170 & 0.089 & 0.290 & 0.200 & 0.140 & 0.270 & 0.200 & 0.062 & 0.041 & 0.088 & 0.079 & 0.036 & 0.220 \\
\hline $\mathrm{Sr}$ & 458.000 & 554.000 & 496.000 & 427.000 & 540.000 & 513.000 & 574.000 & 574.000 & 533.000 & 327.000 & 324.000 & 326.000 & 307.000 & 323.000 & 296.000 \\
\hline $\mathrm{Ba}$ & 352.000 & 543.000 & 395.000 & 384.000 & 478.000 & 543.000 & 444.000 & 465.000 & 501.000 & 473.000 & 501.000 & 506.000 & 368.000 & 481.000 & 445.000 \\
\hline $\mathrm{V}$ & 69.100 & 80.700 & 78.800 & 51.800 & 82.300 & 78.300 & 87.000 & 87.900 & 74.600 & 101.000 & 84.200 & 89.400 & 79.600 & 88.300 & 92.800 \\
\hline
\end{tabular}


Table 2. Cont.

\begin{tabular}{|c|c|c|c|c|c|c|c|c|c|c|c|c|c|c|c|}
\hline Samples & P10(19) & P10(88) & P10(130) & P10(161) & $P 10(x x 1)$ & $P 10(x x 2)$ & P10(xx3) & P10(xx4) & P10(xx5) & P11(6) & P11(8) & P11(18) & P11(20) & P11(34) & P11(41) \\
\hline Lithology & \multicolumn{15}{|c|}{ Quartz Diorite } \\
\hline $\mathrm{Nb}$ & 9.880 & 10.700 & 9.770 & 9.740 & 11.400 & 11.000 & 11.100 & 10.800 & 11.200 & 10.500 & 10.800 & 11.400 & 8.790 & 11.500 & 13.100 \\
\hline $\mathrm{Ta}$ & 0.820 & 0.790 & 0.920 & 0.980 & 0.800 & 0.820 & 0.920 & 0.870 & 0.960 & 0.910 & 0.920 & 0.940 & 0.890 & 1.000 & 1.190 \\
\hline $\mathrm{Zr}$ & 98.000 & 44.400 & 92.200 & 118.000 & 65.000 & 49.900 & 65.300 & 75.000 & 69.900 & 98.900 & 92.500 & 104.000 & 87.800 & 155.000 & 94.600 \\
\hline Hf & 3.230 & 1.420 & 3.070 & 3.700 & 1.920 & 1.670 & 2.060 & 2.350 & 2.000 & 3.500 & 2.820 & 3.370 & 3.360 & 5.590 & 3.190 \\
\hline Sn & 1.890 & 2.250 & 2.010 & 1.920 & 2.500 & 2.170 & 3.470 & 2.570 & 2.420 & 1.660 & 1.600 & 2.100 & 1.750 & 1.820 & 2.250 \\
\hline $\mathrm{Au}$ & 1.080 & 1.390 & 0.720 & 0.250 & 1.700 & 2.020 & 0.510 & 0.880 & 1.700 & 1.130 & 1.760 & 1.380 & 0.380 & 0.760 & 0.510 \\
\hline $\mathrm{Ag}$ & 0.018 & 0.033 & 0.039 & 0.014 & 0.028 & 0.072 & 0.040 & 0.042 & 0.044 & 0.048 & 0.058 & 0.056 & 0.046 & 0.045 & 0.086 \\
\hline Th & 7.770 & 9.660 & 11.200 & 12.000 & 15.400 & 11.900 & 9.220 & 12.500 & 12.400 & 11.700 & 12.800 & 13.900 & 16.200 & 20.100 & 17.400 \\
\hline $\mathrm{Ti}$ & 0.339 & 0.383 & 0.370 & 0.270 & 0.413 & 0.399 & 0.379 & 0.365 & 0.336 & 0.340 & 0.324 & 0.344 & 0.306 & 0.329 & 0.320 \\
\hline $\mathrm{La}$ & 33.200 & 38.400 & 32.200 & 35.600 & 39.000 & 35.800 & 36.100 & 40.400 & 37.000 & 24.800 & 27.600 & 34.200 & 29.900 & 29.600 & 31.200 \\
\hline $\mathrm{Ce}$ & 55.400 & 62.300 & 55.200 & 61.000 & 63.500 & 59.800 & 61.300 & 67.200 & 61.800 & 41.900 & 44.800 & 53.400 & 49.000 & 47.100 & 51.000 \\
\hline $\operatorname{Pr}$ & 6.460 & 8.370 & 6.580 & 7.250 & 8.120 & 7.670 & 8.170 & 8.670 & 8.040 & 5.350 & 5.730 & 7.000 & 5.650 & 5.990 & 6.700 \\
\hline $\mathrm{Nd}$ & 23.500 & 30.600 & 24.300 & 26.200 & 31.200 & 28.900 & 31.900 & 33.400 & 30.100 & 19.700 & 21.400 & 25.200 & 20.100 & 21.900 & 24.400 \\
\hline $\mathrm{Sm}$ & 3.870 & 5.140 & 4.110 & 4.360 & 5.240 & 4.990 & 5.500 & 5.620 & 5.060 & 3.660 & 4.080 & 4.780 & 3.590 & 3.940 & 4.610 \\
\hline $\mathrm{Eu}$ & 1.100 & 1.300 & 1.070 & 1.080 & 1.290 & 1.300 & 1.350 & 1.410 & 1.280 & 0.980 & 0.950 & 1.070 & 0.940 & 0.920 & 0.950 \\
\hline $\mathrm{Gd}$ & 3.230 & 4.340 & 3.300 & 3.550 & 4.340 & 4.200 & 4.600 & 4.720 & 4.150 & 3.380 & 3.660 & 4.310 & 3.170 & 3.450 & 4.040 \\
\hline Dy & 2.230 & 2.650 & 2.380 & 2.320 & 2.740 & 2.710 & 3.080 & 2.940 & 2.660 & 2.960 & 3.230 & 3.580 & 2.700 & 2.850 & 3.340 \\
\hline Ho & 0.420 & 0.490 & 0.430 & 0.440 & 0.520 & 0.480 & 0.570 & 0.540 & 0.480 & 0.580 & 0.620 & 0.700 & 0.560 & 0.570 & 0.650 \\
\hline Er & 1.100 & 1.310 & 1.140 & 1.140 & 1.300 & 1.340 & 1.500 & 1.400 & 1.240 & 1.580 & 1.670 & 1.940 & 1.560 & 1.590 & 1.820 \\
\hline $\mathrm{Tm}$ & 0.160 & 0.190 & 0.170 & 0.160 & 0.190 & 0.180 & 0.210 & 0.200 & 0.180 & 0.240 & 0.260 & 0.310 & 0.240 & 0.270 & 0.310 \\
\hline $\mathrm{Yb}$ & 0.980 & 1.150 & 1.080 & 1.020 & 1.280 & 1.220 & 1.420 & 1.360 & 1.180 & 1.800 & 1.920 & 2.110 & 1.600 & 1.760 & 2.190 \\
\hline $\mathrm{Lu}$ & 0.130 & 0.150 & 0.150 & 0.140 & 0.160 & 0.160 & 0.190 & 0.170 & 0.150 & 0.240 & 0.240 & 0.280 & 0.230 & 0.250 & 0.300 \\
\hline $\mathrm{Y}$ & 9.850 & 11.600 & 10.600 & 10.600 & 12.400 & 11.900 & 13.800 & 12.800 & 11.600 & 14.400 & 15.300 & 17.600 & 14.100 & 14.300 & 16.500 \\
\hline $\mathrm{La} / \mathrm{Yb}$ & 33.878 & 33.391 & 29.815 & 34.902 & 30.469 & 29.344 & 25.423 & 29.706 & 31.356 & 13.778 & 14.375 & 16.209 & 18.688 & 16.818 & 14.247 \\
\hline Sr / Y & 46.497 & 47.759 & 46.792 & 40.283 & 43.548 & 43.109 & 41.594 & 44.844 & 45.948 & 22.708 & 21.176 & 18.523 & 21.773 & 22.587 & 17.939 \\
\hline$\sum \mathrm{REE}$ & 132.210 & 156.950 & 132.550 & 144.720 & 159.440 & 149.270 & 156.510 & 168.640 & 153.850 & 107.660 & 116.710 & 139.500 & 119.710 & 120.690 & 132.080 \\
\hline$\delta \mathrm{Eu}$ & 0.934 & 0.827 & 0.868 & 0.821 & 0.811 & 0.853 & 0.806 & 0.822 & 0.836 & 0.845 & 0.744 & 0.713 & 0.841 & 0.753 & 0.664 \\
\hline$(\mathrm{La} / \mathrm{Yb})_{\mathrm{N}}$ & 22.316 & 21.996 & 19.640 & 22.991 & 20.071 & 19.330 & 16.747 & 19.568 & 20.655 & 9.076 & 9.469 & 10.677 & 12.310 & 11.079 & 9.385 \\
\hline
\end{tabular}



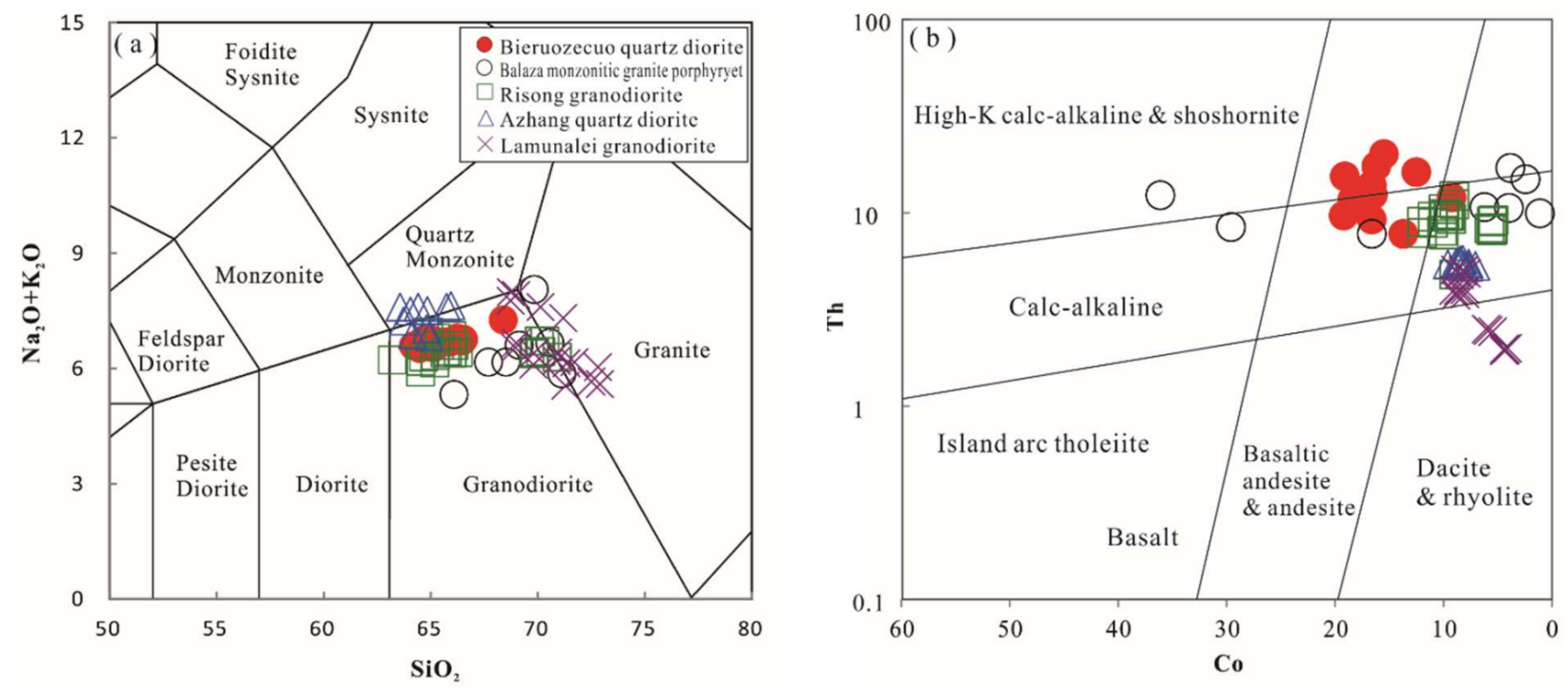

Figure 6. (a) TAS classification and nomenclature diagram [25]; (b) Th-Co diagram showing sample compositional variations, after [26].

The trace and REE composition of the Bieruozecuo quartz diorites are consistent with previously reported Cretaceous adakitic rocks in the Lhasa block (Figure 7), suggesting a possible genetic relationship. All the rocks are characterized by the enrichment of light rare earth elements (LREEs) and depletion of heavy rare earth elements (HREEs) $\left[(\mathrm{La} / \mathrm{Yb})_{\mathrm{N}}=9.08-22.99\right.$, Figure 7a]. The REE contents of the quartz monzonite are much lower than the mean value of the upper crust (210.10 ppm) [27] and display slightly negative $\mathrm{Eu}$ anomalies $\left(\mathrm{Eu} / \mathrm{Eu}^{*}=0.66-0.94\right)$. All rocks exhibit overall enriched large ion lithophile elements (LILE, e.g., $\mathrm{Rb}$ and $\mathrm{K}$ ) and LREE, and are depleted in high field strength elements (HFSE, e.g., $\mathrm{Nb}$, Ti, and P; Figure $7 \mathrm{~b}$ ). The samples show marked $\mathrm{Nb}$ and Ti negative anomalies. $\mathrm{Nb}$ negative anomaly is a feature of the continental crust [28], indicating crustal materials are involved in the magmatic source [29] of the Bieruozecuo quartz diorites. These plutonic rocks also show high Sr content (296.00-574.00 ppm), high $\mathrm{Sr} / \mathrm{Y}$ ratio (17.94-47.76), low Y content (9.85-17.60 ppm) and Yb content (0.98-2.19 ppm), which are comparable with adakitic composition (Figure 8).
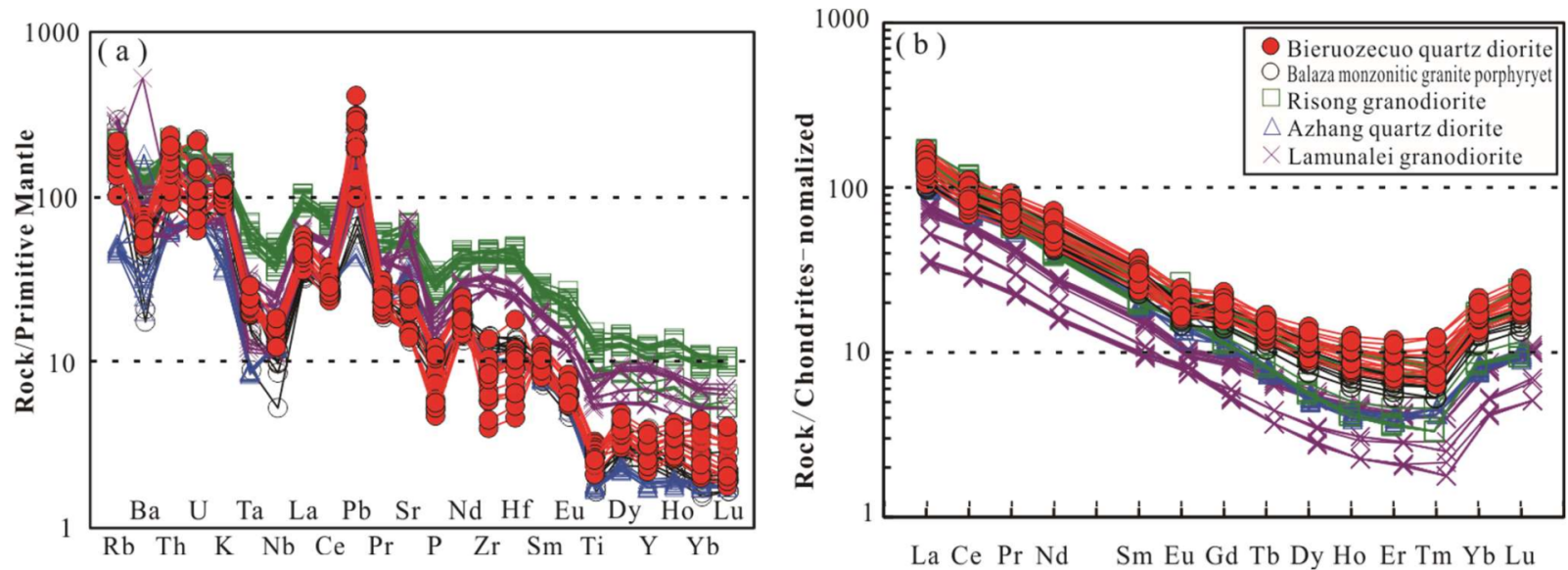

Figure 7. (a) Primitive mantle-normalized trace element spider diagrams (normalization values, after [30]); (b) chondrite-normalized REE patterns (normalization values, after [31]). 

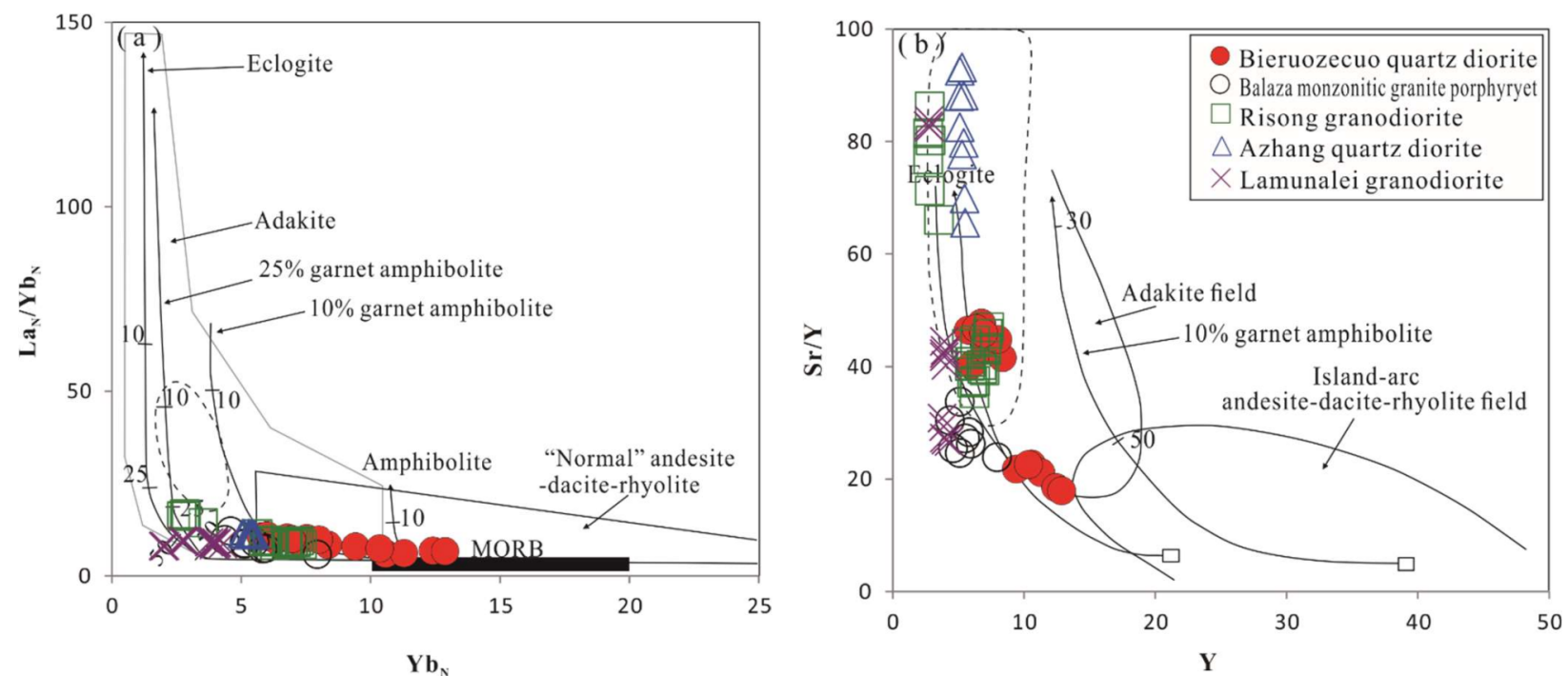

Figure 8. (a) $(\mathrm{La} / \mathrm{Yb}) \mathrm{N}$ vs. $\mathrm{YbN}[32,33]$ and (b) plots of $\mathrm{Sr} / \mathrm{Y}$ vs. $\mathrm{Y}[34]$.

\section{Discussion}

\subsection{Geochemical Classification}

The "adakite" was first proposed by [34], which is considered to be one type of intermediate-felsic igneous rock with special geochemical characteristics. These adakite rocks usually have the following characteristic: $\mathrm{SiO}_{2}>56 \mathrm{wt} . \%, \mathrm{Al}_{2} \mathrm{O}_{3}>15 \mathrm{wt.} \%, \mathrm{MgO}<3 \mathrm{wt} . \%$, $\mathrm{Sr} / \mathrm{Y}$ ratio $(>20$, depleted in $\mathrm{Y}(<18 \mathrm{ppm})$ and $\operatorname{HREE~}(\mathrm{Yb}<19 \mathrm{ppm})$, high $\mathrm{Sr}(>400 \mathrm{ppm}$ or $300 \mathrm{ppm}$ ) (positive or without $\mathrm{Eu}$ ), and $\mathrm{Sr}$ anomalies (depleted in high field strength elements) [33,35-38]. The generation of adakites was initially proposed to be related to the partial melting of the young and hot subducted oceanic lithosphere under eclogite facies condition [34,38], although their origin is much more complex. Many studies show that some igneous rocks with adakite-like geochemical affinities are actually unrelated to slab melting, and they proposed a broad definition of adakites, compassing a wide range of island arc magmatic rocks and collision-related rocks [36,39]. Thus, the term "adakitic" is proposed to distinguish rocks with adakite-like geochemical features to those rocks formed by slab melting [39-41]. At present, many rocks are classified as adakitic, and many studies show that adakitic rocks and typical adakites can be formed in several geodynamic settings, including oceanic subduction, syn-collision, post-collision extension, and within-plate rifting [35-41]; thus, adakitic rocks and typical adakites are one key probe to study the tectonic evolution of paleo-orogenic belt.

The studied Bieruozecuo quartz diorites samples have high contents of $\mathrm{SiO}_{2}$ (64.07-68.32 wt.\%) and $\mathrm{Al}_{2} \mathrm{O}_{3}(14.72-15.75 \mathrm{wt} . \%)$, with high $\mathrm{Sr} / \mathrm{Y}(21.18-47.76)$ and $\mathrm{La} / \mathrm{Yb}$ (13.78-34.90), as well as low $\mathrm{MgO}(1.60-2.67 \mathrm{wt} . \%), \mathrm{Y}(9.850-16.500 \mathrm{ppm})$, and $\mathrm{Yb}$ $(0.980-2.190 \mathrm{ppm})$. The geochemical discrimination diagrams of $(\mathrm{La} / \mathrm{Yb})_{\mathrm{N}} \mathrm{vs}$. $\mathrm{Yb}_{\mathrm{N}}$ and $\mathrm{Sr} / \mathrm{Y}$ vs. Y show that the studied Bieruozecuo quartz diorites exhibits typical adakitic geochemical affinities (Figure 8). Previous studies have reported several ca. 90 82 Ma adakitic plutons in the north and central Lhasa blocks. The Bieruozecuo quartz diorites studied in this paper has similar geochemical composition and crystallization age to those reported adakitic plutons (Figures 5-8), suggesting that the adakitic magmatic activity in the north and central Lhasa blocks may last from $116 \mathrm{Ma}$ to $82 \mathrm{Ma}$.

\subsection{Petrogenesis}

Numerous studies show that the petrogenesis of intermediate igneous rocks is complex and various, including the mixing of mantle-derived mafic magma and granitic magma, partial melting of mafic crust, and AFC of mantle-derived magma [42-44]. The Early Cretaceous Bieruzecuo quartz diorites are meta-aluminous high-K calc-alkaline plutonic rock, with high $\mathrm{K}_{2} \mathrm{O}$ (average content of 3.13 wt.\%) and low $\mathrm{CaO}$ (average content of 
$4.16 \mathrm{wt} . \%)$, indicating that the magma mainly originated from the partial melting of continental crust [45]. The trace elements of $\mathrm{Cr}$ (20.8-63.5 ppm, with an average of $40.9 \mathrm{ppm})$, $\mathrm{Ni}$ (10.4-32.0 ppm, with an average of 21.4 ppm), and Co (9.3-19.2 ppm, with an average of $16.1 \mathrm{ppm})$ are very close to the continental crust, and the $\mathrm{La} / \mathrm{Nb}$ ratios (2.38-3.74) are significantly different from mantle-derived magma $(\mathrm{La} / \mathrm{Nb} \approx 0.96)[30] . \mathrm{Nb}$ and $\mathrm{Ta}$ are stable in the process of erosion and metamorphism and have the characteristics of tracing magma origin $[46,47]$. The $\mathrm{Nb} / \mathrm{Ta}$ ratios of the Late Early Cretaceous quartz diorite is 9.88-13.54, which is close to the average crustal value (12-13) but significantly lower than the mantle magma value (17 $\pm 1,[48])$. The $\mathrm{Nd} / \mathrm{Th}$ ratios (1.09-3.46) are consistent with the $\mathrm{Nd} / \mathrm{Th}$ ratio for the crustal source $(\approx 3)$ and significantly lower than that of the mantle source (>15) [49], suggesting that the magma may not derive from partial melting of mantle peridotite or separation and crystallization of basaltic magma [50], but from partial melting of continental crustal rocks [51]. The Ti/Zr ratios (21.23-86.26, average 44.97) and $\mathrm{Ti} / \mathrm{Y}$ ratios (193.94-349.06, average 272.02) are slightly higher than those of continental crust rocks $(\mathrm{Ti} / \mathrm{Zr}<30, \mathrm{Ti} / \mathrm{Y}<200)$ [52], which may be related to the presence of garnet residues in the source [53] and resemblance with the high $\mathrm{La} / \mathrm{Yb}$ and $\mathrm{Sr} / \mathrm{Y}$ ratios of rocks.

The Bieruzecuo quartz diorites have the geochemical characteristics of adakitic rocks. According to previous works, the genesis of adakitic rocks may be due to: (1) partial melting of young oceanic crust [34,40,54-56]; (2) fractional crystallization of primitive basaltic magma and assimilation with crustal materials, i.e., the AFC model [34,57]; (3) magma mixing between felsic and mafic magmas [58,59]; (4) partial melting of a thickened lower crust or delaminated lower crust under high-temperature conditions [38,60,61]; and (5) partial melting of subducted continental crust $[38,61]$. The Bieruzecuo adakitic rocks have high $\mathrm{SiO}_{2}$ content (65-69.32 wt.\%); there is no obvious $\mathrm{Eu}$ anomaly in the REE patterns, and the rocks do not show assemblages of gabbro-diorite-granodiorite, which precludes the AFC model of the geochemical trends in $\mathrm{Rb} / \mathrm{Nd}$ vs. $\mathrm{Rb}$, and the $\mathrm{La} / \mathrm{Sm}$ vs. Sm diagrams do not support the AFC model (Figure 9). As mentioned above, the Bieruzecuo quartz diorites are mainly derived from the partial melting of continental crust materials, which is consistent with their high ratios of $\mathrm{K}_{2} \mathrm{O} / \mathrm{Na}_{2} \mathrm{O}$ (Figure 10A).

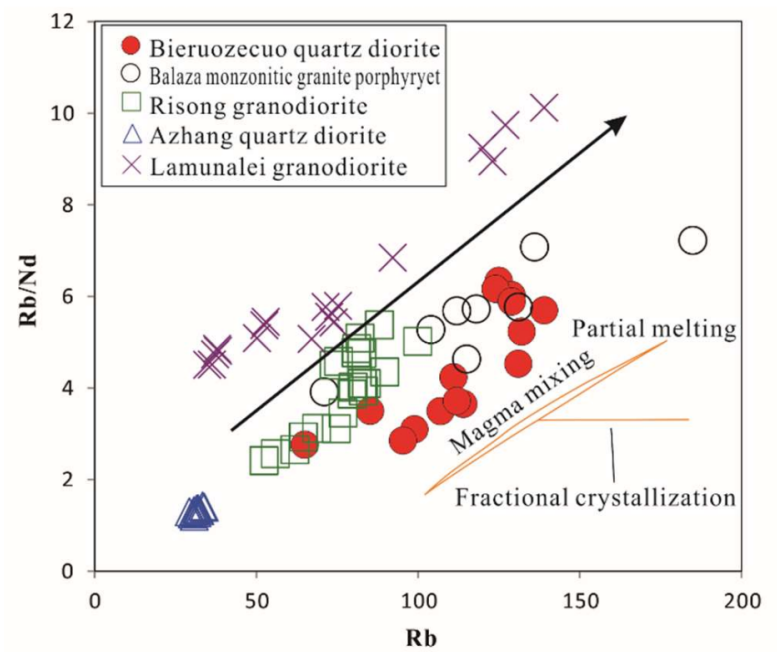

(a)

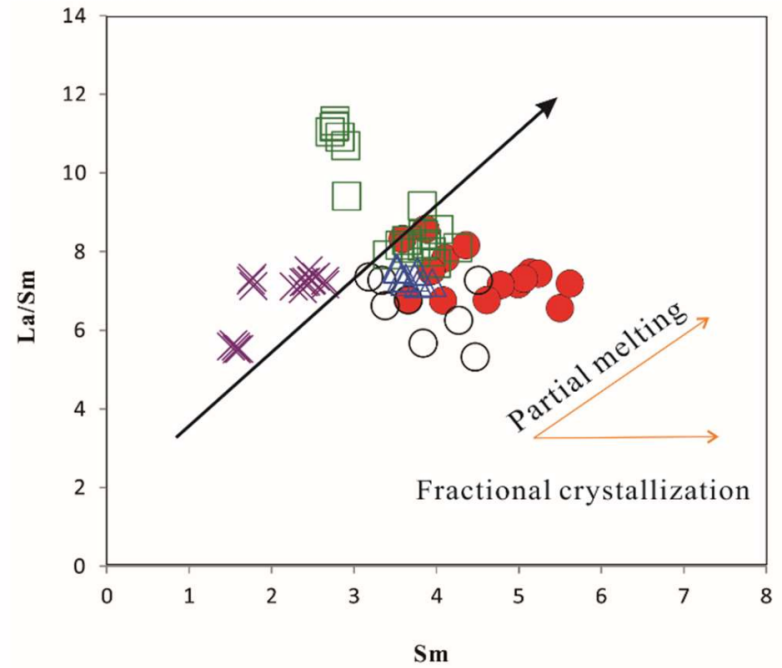

(b)

Figure 9. Discrimination diagrams of petrogenetic processes of the Bieruozecuo quartz diorites. (a) $\mathrm{Rb} / \mathrm{Nd}$ s. $\mathrm{Rb}$ (after [62]); (b) La/Sm s. La (after [63]).

Based on the geochemical characteristics of the Bieruozecuo adakites, we propose that the studied adakites may be the product of partial melting of the delaminated lower crust. In general, the adakite formed by partial melting of the delaminated lower crust is easy to modify via the addition of mantle materials during magma ascending and emplacement, resulting in high $\mathrm{MgO}, \mathrm{Cr}$, and $\mathrm{Ni}$ contents in the rocks $[21,40,64,65]$. The studied adakites 
have high Mg\#, Cr (20.80-63.50 ppm), and Ni (10.40-32.00 ppm), resembling the rocks that derived from the melting of the delaminated lower crust or subducted oceanic crust, but differ from the adakite formed by partial melting of the thickened lower crust (Figure 10). However, the geochemical features of the high ratios of $\mathrm{K}_{2} \mathrm{O} / \mathrm{Na}_{2} \mathrm{O}$ and low ratios of $\mathrm{CaO} / \mathrm{Al}_{2} \mathrm{O}_{3}$ strongly exclude the model of the melting of the subducted oceanic crust (Figure 10a). Thus, the melting of the delaminated continental lower crust is proposed to be the most possible model for the origin of the studied adakitic rocks in the northern Lhasa block.
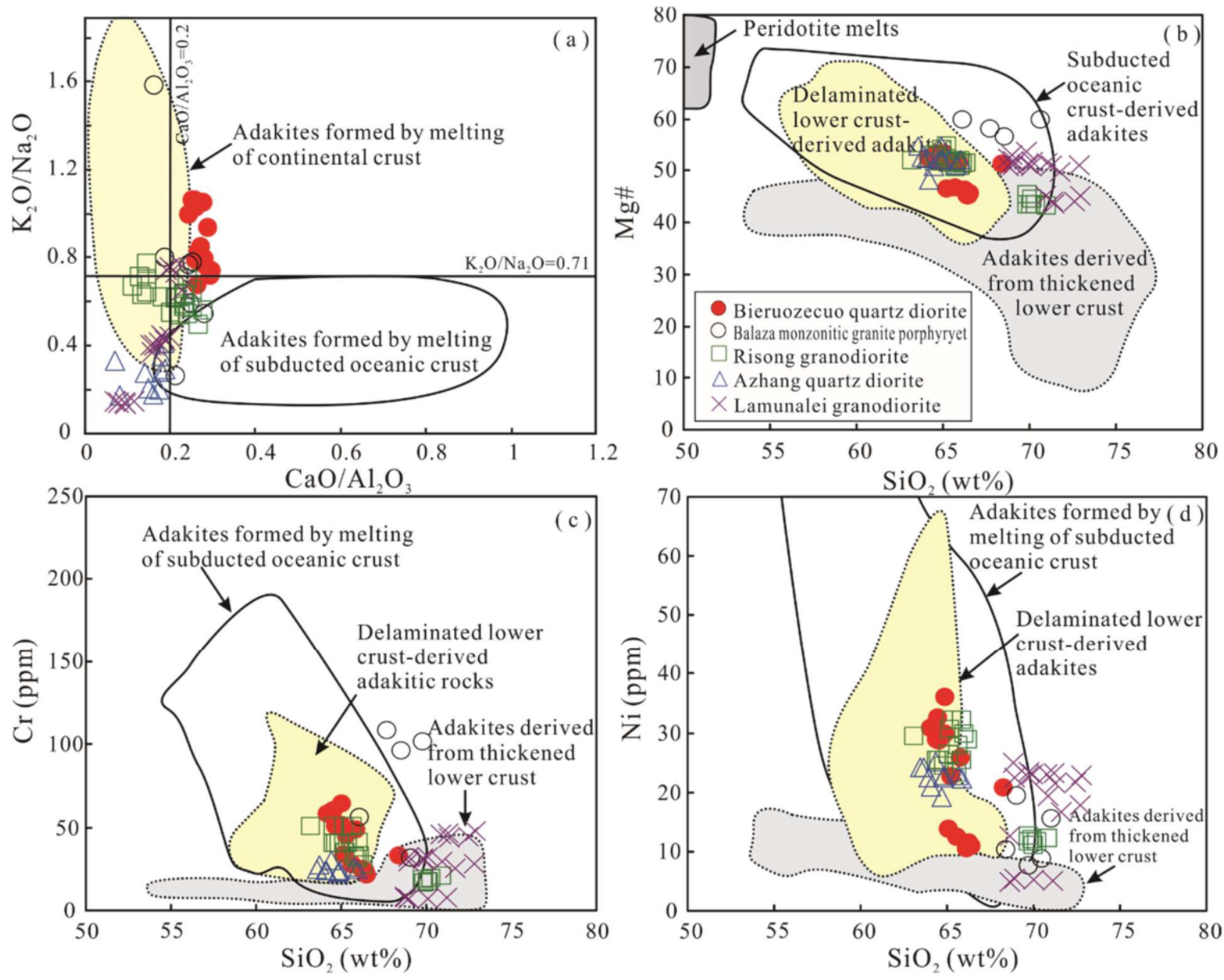

Figure 10. Relationship diagram of geochemical characteristics of the major elements for the Bieruozecuo adakitic rocks. Diagram (a) after [66]; (b) after [67]; (c) after [68]; (d) after [68].

\subsection{Tectonic Setting and Geological Significance}

Previous studies show that the Tethyan ophiolites, along the BNSZ, are overlain unconformably by clastic sequences of the Lower Cretaceous Dongqiao Formation (ca. 130-118 Ma) $[7,9,14]$. This relationship suggests that the BNSZ ocean basin was closed in the Early Cretaceous, and collision between north Lhasa and Qiangtang blocks occurred at the Early Cretaceous [69]. However, the transition from syn-collision to post-collisional extension is still unclear, resulting in the discussion of the geodynamic setting of the Cretaceous magmatism in the Lhasa block.

As shown in Figure 1, the Early Cretaceous is an important magmatic activity period in the Lhasa block, which occurs almost simultaneously in the south (ca. 100-137 Ma), the central (ca. 95-145 Ma), and north Lhasa blocks (ca. 110-133 Ma). The peak period of magmatic activity is concentrated in ca. $120-110 \mathrm{Ma}[2,70]$, and the Bieruozecuo adakitic quartz diorite is the product of this peak magmatism. Recent studies have shown that the collision between the Lhasa and Qiangtang blocks in Early Cretaceous may result in the thickening of the continental crust of the north Lhasa block [7,69]. As shown in Figure 11, 
The Bieruzecuo quartz diorites mostly fall in the field of volcanic arc granites (VAG). The ca. 116-114 Ma Bieruozecuo quartz diorite has the geochemical characteristics identical to magmas originating from delaminated crust, which indicates, once again, that the largescale adakitic magmatism in the northern and central Lhasa blocks is the response to the delamination of continental lower crust, as well as the tectonic transition from syn-collision to post-collisional extension, occurring at ca. 120-110 Ma.

Therefore, we propose that the Bieruzecuo pluton is the product of post-collisional extension and derived from the partial melting of the delaminated continental lower crust. Continental crust delamination may be responsible for the post-collisional extension, which is an important mechanism to the ca. 120-110 Ma adakitic magmatism flare-up event in the Lhasa block (Figure 12).
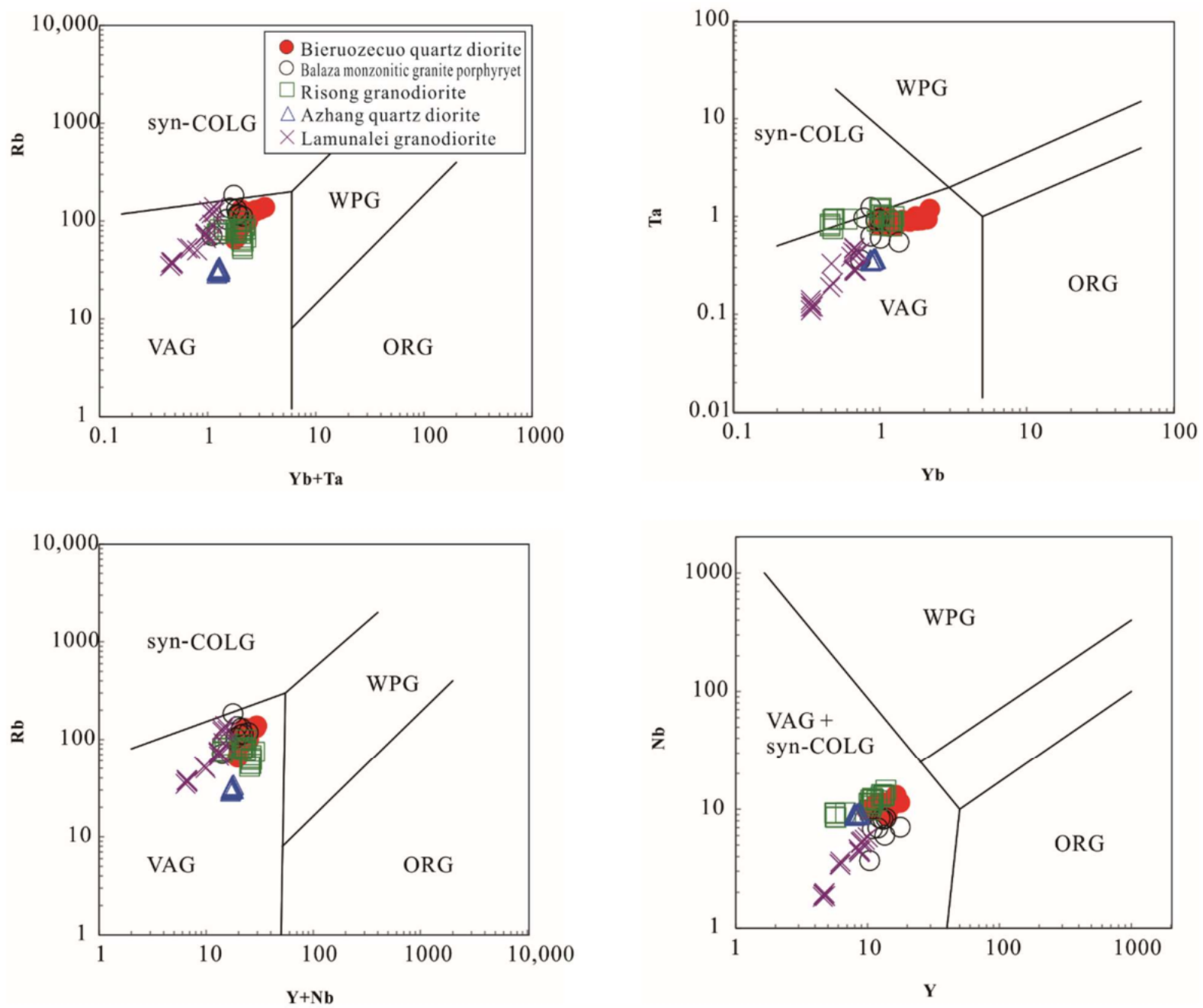

Figure 11. Bulk geochemical data for the Bieruozecuo adakitic rock from the Lhasa block, Tibet, plotted on the Pearce diagram [71]. 


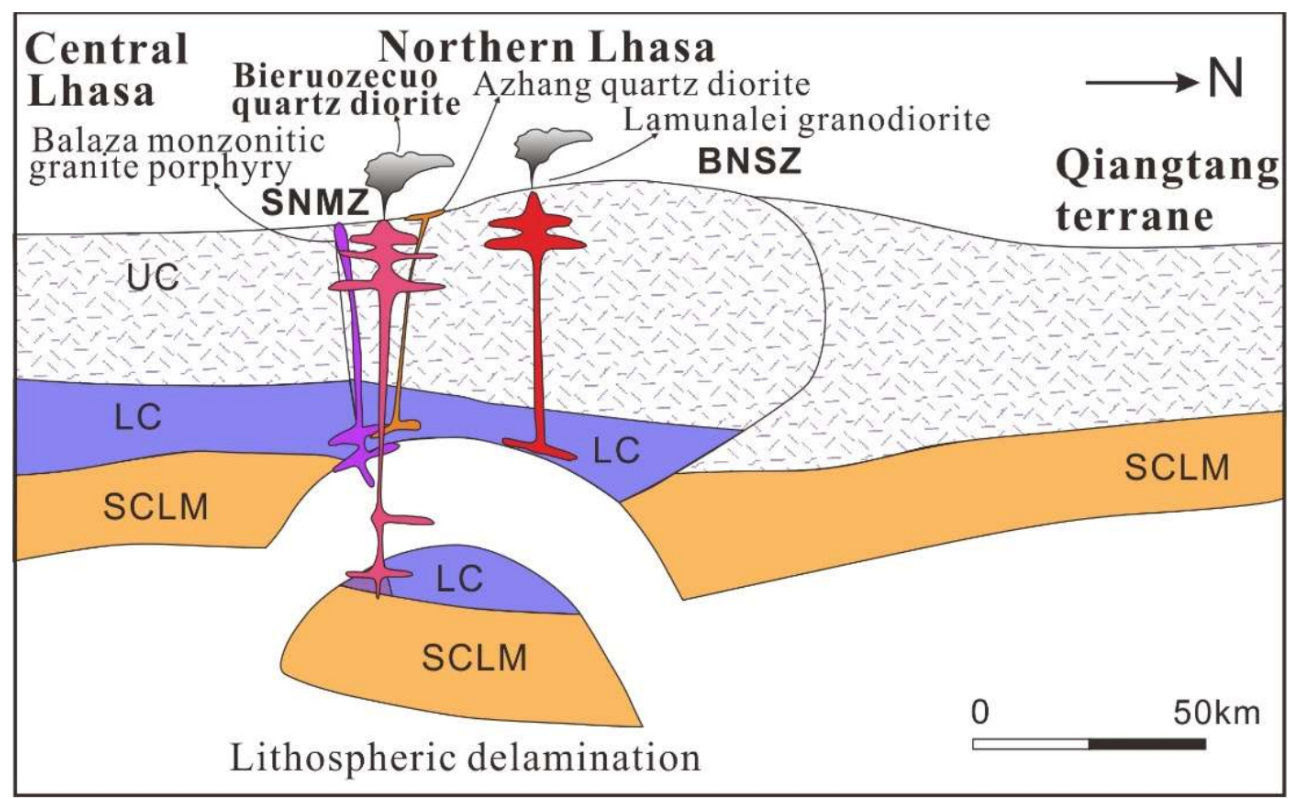

Figure 12. Schematic illustration showing the geodynamic evolution of the central-northern Lhasa subterrane, at ca. 120-110 Ma. BNSZ = Bangong-Nujiang suture zone; SNMZ = Shiquan River-Nam Tso mélange zone; UC= upper crust; LC = lower crust; and SCLM = sub-continental lithospheric mantle.

\section{Conclusions}

(1) LA-ICP-MS zircon U-Pb geochronology shows that the Bieruozecuo quartz diorite from north Lhasa block crystallized at ca. 116-114 Ma (Early Cretaceous magmatic event).

(2) The Bieruozecuo quartz diorite was enriched in $\mathrm{Al}_{2} \mathrm{O}_{3}$ and $\mathrm{Na}_{2} \mathrm{O}$, but relatively depleted in $\mathrm{TiO}_{2}, \mathrm{MgO}$, and $\mathrm{MnO}$, which may be included in the quasi-alumina high$\mathrm{K}$ calc-alkaline series. They display the geochemical characteristics typical of adakitic rocks, with high $\mathrm{Sr}$, low $\mathrm{Y}$ and $\mathrm{Yb}$, as well as high $\mathrm{Sr} / \mathrm{Y}$ and $\mathrm{La} / \mathrm{Yb}$ ratios. The high $\mathrm{Mg \# \text {, }}$ $\mathrm{K}_{2} \mathrm{O} / \mathrm{Na}_{2} \mathrm{O}$ ratios, $\mathrm{Cr}$, and $\mathrm{Ni}$ contents indicate that these adaktic rocks probably derived from the partial melting of the delaminated continental crust, without obvious fractional crystallization during magma ascending and emplacement.

(3) The Early Cretaceous flare-up event of adaktic magmatism of the Lhasa block is a response to the delamination of continental crust, as well as the transition from syn-collision to post-collisional extension at ca. 120-110 Ma.

Author Contributions: Conceptualization, Q.-R.X. and Y.-F.W.; methodology, F.-H.X.; software, Z.-Y.L.; formal analysis, Q.-R.X., Y.-F.W., and Z.-Y.L.; investigation, Y.-F.W. and Z.-Y.L.; resources, Y.-F.W.; data curation, Q.-R.X. and J.-X.W.; writing—original draft preparation, Q.-R.X.; writingreview and editing, F.-H.X. and L.F.; visualization, Q.-R.X., L.F., and H.Z.; project administration, Y.-F.W.; funding acquisition, Y.-F.W. All authors have read and agreed to the published version of the manuscript.

Funding: This work was supported by the project, named Geological and Mineral Survey, of Gzezao Bieruozuo, 1:50,000, in Tibet (I44E022020, I44E022021, I44E023020, I44E023021).

Data Availability Statement: The data supporting reported results can be found in the text.

Acknowledgments: We gratefully acknowledge the research members, who are Zeng Wei, Shao Zhiwei, Yu Xuhui, Tang Guangcheng, Zhu Guobao, etc., for their cooperation and help.

Conflicts of Interest: The authors declare no conflict of interest. 


\section{References}

1. Mo, X.X.; Dong, G.C.; Zhao, Z.D.; Zhou, S.; Wang, L.L.; Qiu, R.Z.; Zhang, F.Q. Spatial and Temporal Distribution and Characteristics of Granitoids in the Gangdese, Tibet and Implication for Crustal Growth and Evolution. Geol. J. China Univ. 2005, 11, 281-290.

2. Zhu, D.C.; Pan, G.T.; Mo, X.X.; Wang, L.Q.; Liao, Z.L.; Zhao, Z.D.; Dong, G.C.; Zhou, C.Y. Late Jurassic-Early Cretaceous geodynamic setting in middle-northern Gandese: New insights from volcanic rocks. Acta Petrol. Sin. 2006, 22, 534-546.

3. Zhu, D.C.; Pan, G.T.; Wang, L.Q.; Mo, X.X.; Zhao, Z.D.; Zhou, C.Y.; Liao, Z.L.; Dong, G.C.; Yuan, S.H. Spatial-temporal distribution and tectonic setting of Jurassic magmatism in the Gangdise belt, Tibet, China. Geol. Bull. China 2008, 4, 458-468.

4. Ma, G.L.; Yue, Y.H. Cretaceous volcanic rocks in northern Lhasa block: Constraints on the tectonic evolution of the Gangdese arc Acta Petrol. Mineral. 2010, 29, 525-538. (In Chinese)

5. Wu, Z.H.; Wu, X.W.; Zhao, Z.; Lu, L.; Ye, P.S.; Zhang, Y.L. Shrimp U-Pb isotopic dating of the Late Cretaceous volcanic rocks and its chronological constraint on the red-beds in southern Qiangtang Block. Acta Geosci. Sin. 2014, 35, 567-572. (In Chinese)

6. Zhang, S.; Shi, H.F.; Hao, H.J.; Li, D.W.; Lin, Y.; Feng, M.X. Geochronology, geochemistry and tectonic significance of Late Cretaceous adakites in Bangong Lake, Tibet. Earth Sci. (J. China Univ. Geosci.) 2014, 39, 509-524, (In Chinese with English abstract).

7. Kapp, P.; DeCelles, P.G.; Gehrels, G.E.; Heizier, M.; Ding, L. Geological records of the Lhasa-Qiangtang and Indo-Asian collisions in the Nima area of central Tibet. Geol. Soc. Am. Bull. 2007, 119, 917-933. [CrossRef]

8. Wen, D.R.; Liu, D.Y.; Chung, S.L.; Chu, M.F.; Ji, J.Q.; Zhang, Q.; Song, B.; Lee, T.Y.; Yeh, M.W.; Lo, C.H. Zircon SHRIMP U-Pb ages of the Gangdese Batholith and implications for Neotethyan subduction in southern Tibet. Chem. Geol. 2008, 252, 191-201. [CrossRef]

9. Zhu, D.C.; Mo, X.X.; Niu, Y.L.; Zhao, Z.D.; Wang, L.Q.; Liu, Y.S.; Wu, F.Y. Geochemical investigation of Early Cretaceous igneous rocks along an east-west traverse throughout the central Lhasa Terrane, Tibet. Chem. Geol. 2009, 268, 298-312. [CrossRef]

10. Zhu, D.C.; Zhao, Z.D.; Niu, Y.L.; Mo, X.X.; Chung, S.L.; Hou, Z.Q.; Wu, F.Y. The Lhasa Terrane: Record of a microcontinent and its histories of drift and growth. Earth Planet. Sci. Lett. 2011, 301, 241-255. [CrossRef]

11. Powell, C.M.; Conaghan, P.J. Plate tectonics and the Himalayas. Earth Planetaru Sci. Lett. 1973, 20, 1-12. [CrossRef]

12. Molnar, P.; Tapponnier, P. Cenozoic tectonice of Asia: Effects of a continental collision: Features of recent continental tectonics in Asia can be interpreted as results of the India-Eurasia collision. Science 1975, 189, 419-426. [CrossRef] [PubMed]

13. Pan, G.T.; Chen, Z.L.; Li, X.Z.; Yan, Y.J.; Xu, X.S.; Xu, Q.; Jiang, X.S.; Wu, Y.L.; Luo, J.N.; Zhu, T.X.; et al. Geological-Tectonic Evolution in the Eastern Tethys; Grological Publishing House: Beijing, China, 1997; pp. 1-218. (In Chinese)

14. Yin, A.; Harrison, T.M. Geologic evolution of the Himalayan-Tibetan Orogen. Annu. Rev. Earth Planet. Sci. 2000, 28, 211-280. [CrossRef]

15. Xu, Z.Q.; Yang, J.S.; Li, W.C.; Li, H.Q.; Cai, Z.H.; Yan, Z.; Ma, C.Q. Paleo- Tethys system and accretionary orogeny in the Tibet Plateau. Acta Petrol. Sin. 2013, 29, 1847-1860, (In Chinese with English abstract).

16. Mo, X.X.; Niu, Y.L.; Dong, G.C.; Zhao, Z.D.; Hou, Z.Q.; Zhou, S.; Ke, S. Contribution of syncollisional felsic magmatism to continental crus growth: A case study of the Paleogene Linzizong volcanic succession in southern Tibet. Chem. Geol. 2008, 250, 49-67. [CrossRef]

17. Wang, Z.Z.; Liu, D.; Zhao, Z.D.; Yan, J.J.; Shi, Q.S.; Mo, X.X. The Sangri highly fractionated I-type granites in southern Gangdese: Petrogenesis and dynamic implication. Acta Petrol. Sin. 2017, 22, 2479-2493. (In Chinese)

18. Wu, F.Y.; Ji, W.Q.; Liu, C.Z.; Chung, S.L. Detrital zircon UePb and Hf isotopicdata from the Xigaze fore-arc basin: Constraints on Transhimalayan. Chem. Geol. 2010, 271, 13-25. [CrossRef]

19. Huang, S.H.; Chen, J.L.; Zen, Y.C.; Tan, R.Y.; Huang, T.T.; Liu, X.J. Geochemical characteristics of Early Cretaceous magmatic rocks in the northwestern part of the Lhasa Block and indications of early crustal growth of the southern plateau. Geochimica 2020 $49,21-35$.

20. Sun, G.Y.; Hu, X.M.; Zhu, D.C.; Hong, W.T.; Wang, J.G.; Wang, Q. Thickened juvenile lower crust-derived 90 Ma adakitic rocks in the central Lhasa terrane, Tibet. Lithos 2015, 224, 225-239. [CrossRef]

21. Yu, H.X.; Chen, J.L.; Xu, J.F.; Wang, B.D.; Wu, J.B.; Liang, H.Y. Geochemistry and origin of Late Cretaceous( 90 Ma) ore-bearing porphyry of Balazha in mid-northern Lhasa terrane, Tibet. Acta Petrol. Sin. 2011, 27, 2011-2022, (In Chinese with English abstract).

22. Dong, Y.C.; Wang, M.; Xie, C.M.; Yu, Y.P.; Hao, Y.J. Genesis and tectonic indication of the Late Cretaceous adakite rocks in the Lamunale area, Nima County, Tibet. Acta Petrol. Sin. 2020, 36, 426-442.

23. Hou, K.J.; Li, Y.H.; Tian, Y.R. In situ U-Pb zircon dating using laser ablation-multi ion counting-ICP-MS. Miner. Depos. 2009, 28, 481-492.

24. Zhang, X.F.; Li, Y.G.; Cao, X.M.; Qu, X.M.; Xin, H.B.; Wang, J.; Du, D.D. LA-ICP-MS zircon U-Pb age and geochemical characteristics of the acid intrusive rocks in the western part of Bangong Lake-Nujiang River suture zone. Geol. Bull. CHN. 2014, 33, 984-994, (In Chinese with English abstract).

25. Middlemost, E.A.K. Naming materials in the magma/igneous rock system. Earth-Sci. Rev. 1994, 37, 215-224. [CrossRef]

26. Hastie, A.R.; Kerr, A.C.; Pearce, J.A.; Mitchell, S.F. Classification of altered volcanic island arc rocks using immobile trace elements: Development of the Th-Co discrimination diagram. J. Petrol. 2007, 48, 2341-2357. [CrossRef]

27. Maniar, P.D.; Piccoli, P.M. Tectonic discrimination of granitoids. Geol. Soc. Am. Bull. 1989, 101, 635-643. [CrossRef]

28. Jiang, Y.H.; Yang, W.Z. Geochemistry of early Yanshanian granitoids and its tectonic significance in the western Qinghai-Tibet plateau. Mineral. Petrol. 2000, 20, 74-79. 
29. Zhong, H.M.; Tong, J.S.; Lu, R.K.; Xia, J. Geochemical characteristics and tectonic setting of peraluminous granite in the Songxi area, Rutog County, Tibet, China. Geol. Bull. China 2006, 25, 183-188.

30. Sun, S.S.; McDonough, W.F. Chemical and isotopic systematics of oceanic basalts: Implications for mantle composition and processes. In Magmatism in Occean Basins; Sander, A.D., Norry, M.H., Eds.; Geological Society of London Special Publications: London, UK, 1989; Volume 42, pp. 313-345.

31. Taylor, S.R.; McLennan, S.M. The Continental Crust: Its Composition and Evolution; U.S. Department of Energy: Washington, DC, USA, 1985.

32. Drummond, M.S.; Defant, M.J. A model for trondhjemite-tonalite-dacite genesis and crustal growth via slab melting: Archaean to modern comparisons. J. Geophys. Res. 1990, 95, 21503-21521. [CrossRef]

33. Martin, H. Adakitic magmas: Modern analogues of Archaean granitoids. Lithos 1999, 46, 411-429. [CrossRef]

34. Defant, M.J.; Drummond, M.S. Derivation of some modern are magmas by melting of young subducted lithosphere. Nature 1990, 327, 662-665. [CrossRef]

35. Clemens, J. D S-type granitic magmas: Petrgenetic issues, models and evidene. Earth-Sci. Rev. 2003, 61, 1-18. [CrossRef]

36. Castillo, P.R. An overview of adakite petrogenesis. Chin. Sci. Bull. 2006, 51, 257-268. [CrossRef]

37. Castillo, P.R. Adakite petrogenesis. Lithos 2012, 134,135, 304-316. [CrossRef]

38. Wang, Q.; Xu, J.F.; Zhao, Z.H.; Zi, F.; Tang, G.J.; Jia, X.H.; Jiang, Z.Q. Tectonic setting and associated rock suites of adakitc rocks. Bull. Mineral. Petrol. Geochem. 2008, 27, 344-350. (In Chinese)

39. Moyen, J.F. High Sr/Y and La/Yb ratios: The meaning of the "adakitic signature". Lithos 2009, 112, 556-574. [CrossRef]

40. Martin, H.; Smithies, R.H.; Rapp, R.; Moyen, J.F.; Champion, D. An overview of adakite, tonalite-trondhjemite-granodiorite(TTG), and sanukitoid: Relationships and some implications for crustal evolution. Lithos 2005, 79, 1-24. [CrossRef]

41. Xu, J.F.; Wu, J.B.; Wang, Q.; Chen, J.L.; Cao, K. Research advances of adakites and adakitic rocks in China. Bull. Mineral. Petrol. Geochem. 2014, 33, 6-13, (In Chinese with English abstract).

42. Arnaud, N.O.; Vidal, P.H.; Tapponnier, P.; Matte, P.H.; Deng, W.M. The high $\mathrm{K}_{2} \mathrm{O}$ volcanism of northwestern Tibet: Geochemistry and tectonic implications. Earth Planet. Sci. Lett. 1992, 111, 351-367. [CrossRef]

43. Thompson, A.B. Fertility of crusta1 rocks during anatexis. Trans. R. Soc. Edinburgh Earth Sci. 1996, 87, 1-10.

44. Wang, Y.J.; Fan, W.M.; Guo, F. Geochemistry of early Mesozoic potassium-rich diorites-granodiorites in southeastern Hunan Province, South China: Petrogenesis and tectonic implications. Geochem. J. 2003, 37, 427-448. [CrossRef]

45. Xiao, Q.H.; Deng, J.F.; Ma, D.Q.; Hong, D.W.; Mo, X.X.; Lu, X.X.; Li, Z.C.; Wang, X.W.; Ma, C.Q.; Wu, F.Y.; et al. Thinking and Method of Granite Research; Geological Publishing House: Beijing, China, 2002; pp. 12-20.

46. Barth, M.G.; McDonough, W.; Rudnick, R. Tracking the budget of $\mathrm{Nb}$ and $\mathrm{Ta}$ in the continental crust. Chem. Geol. 2000, 165, 197-213. [CrossRef]

47. Pfänder, J.A.; Münker, C.; Stracke, A.; Mezger, K. Nb/Ta and Zr/Hf in ocean island basalts - implications for crust-mantle differentiation and the fate of Niobium. Earth Planet. Sci. Lett. 2007, 254, 158-172. [CrossRef]

48. Green, T.H. Significance of $\mathrm{Nb} / \mathrm{Ta}$ as an indicator of geochemical processes in the crust-mantle system. Chem. Geol. 1995, 120, 347-359. [CrossRef]

49. Bea, F.; Arzamastsev, A.; Montero, P.; Arzamastseva, L. Aonmalous alkaline rocks of Soustov, Kola:evidence of mantle-derived matasomatic fluids affecting crustal materials. Contrib. Min. Pet. 2001, 140, 554-566. [CrossRef]

50. Li, S.G.; Nie, Y.H.; Zhen, S.G. Interaction between subducted continental crust and upper mantle-I. Geochemistry of major elements and trace elements in the synchromatic mafic-ultramafic rocks in Dabieshan. China Sci. 1997, 27, 488-493.

51. Xie, L.; Dun, D.; Zhu, L.D.; Ni, M.C.; Yang, W.G.; Tao, G.; Li, C.; He, B.; He, Y. Zircon U-Pb geochronology, geochemistry and geological significance of the Zhaduding A-type granites in northern Gangdise, Tibet. Geol. China 2015, 42, 1214-1227.

52. Wedepohl, K.H. The composition of the continental crust. Geochim. Cosmochim. Acta 1995, 59, 1217-1232. [CrossRef]

53. Zhang, Z.Z.; Gu, L.X.; Wu, C.Z.; Zhai, J.P.; Wang, C.S.; Tang, J.H.; Xiao, E. Early-Middle Indosinian Weiya Quartz Diorite, Eastern Segment of the Middle Tianshan Mountains, NW China: Implications for Intra-Continent Subduction and Partial Melting of Juvenile Lower Crust. Acta Geol. Sin. 2011, 85, 1420-1434.

54. Rapp, R.P.; Shimizu, N.; Norman, M.D.; Applegate, G.S. Reaction between slab-derived melts and peridotite in the mantle wedge: Experimental constraints at 3.8GPa. Chem. Geol. 1999, 160, 335-356. [CrossRef]

55. Zhang, Z.M.; Zhao, G.C.; Santosh, M.; Wang, J.L.; Dong, X.; Shen, K. Late Cretaceous charnockite with adakitic affinities from the Gangdese batholith, shoutheastern Tibet: Evidence for Neo-Tethyan mid-ocean ridge subdution? Gondwana Res. 2010, 17, 615-631. [CrossRef]

56. Eyuboglu, Y.; Chung, S.L.; Santosh, M.; Dudas, F.O.; Akaryali, E. Transition from shoshonitic to adakitic magmatism in the eastern Pontides, NE Turkey: Implications for slab window melting. Gondwana Res. 2011, 19, 413-429. [CrossRef]

57. Kang, Z.Q.; Xu, J.F.; Wang, B.D.; Dong, Y.H.; Wang, S.Q.; Chen, J.L. Geochemistry of Cretaceous volcanic rocks of Duoni Formation in northern Lhasa Block: Discussion of tectonic setting. Earth Sci. (J. China Univ. Geosci.) 2009, 34, 89-104. (In Chinese)

58. Guo, F.; Nakamuru, E.; Fan, W.; Kobayoshi, K.; Li, C. Generation of Palaeocene adakitic andesites by magma mixing in Yanji Area, NE China. J. Petrol. 2007, 48, 661-692. [CrossRef]

59. Streck, M.J.; Leeman, W.P.; Chesley, J. High-magnesian andesite from Mount Shasta: A product of magma mixing and contamination, not a primitive mantle melt. Geology 2007, 35, 351-354. [CrossRef] 
60. Wang, Q.; McDermott, F.; Xu, J.F.; Bellon, H.; Zhu, Y.T. Cenozoic K-rich adakitic volcanic rocks in the Hohxil area, northern Tibet: Lower-crustal melting in an intracontinental setting. Geology 2005, 33, 465-468. [CrossRef]

61. Lai, S.C.; Qin, J.F. Adakitic rocks derived from the partial melting of subducted continental crust: Evidence from the Eocene volcanic rocks in the northern Qiangtang block. Gondwana Res. 2013, 23, 812-824. [CrossRef]

62. Schiano, P.; Monzier, M.; Eissen, J.P.; Martin, H.; Koga, K.T. Simple mixing as the major control of the evolution of volcanic suites in the Ecuadorian Andes. Contrib. Mineral. Petrol. 2010, 160, 297-312. [CrossRef]

63. Wu, H.; Li, C.; Xu, M.J.; Li, X.K. Early cretaceous adakitic magmatism in the Dachagou Area, northern Ihasa terrane, Tibet: Implications for slab roll-back and subsequent slab break-off of the lithosphere of the Bangong-Nujiang Occan. J. Asian Earth Sci. 2015, 97, 51-66. [CrossRef]

64. Smithies, R.H. The Archaean tonalite-tonalite-trondhjemite-granodiorite (TTG) series is ont an analogue of Cenozoic adakite. Earth Planetaru Sci. Lett. 2000, 182, 115-125. [CrossRef]

65. Prouteau, G.; Scaillet, B.; Pichabant, M.; Maury, R. Evidence for mantle metasomatism by hydrous silicic melts derived from subducted oceanic crust. Nature 2001, 410, 197-200. [CrossRef] [PubMed]

66. Chen, J.; Wei, J.; Fu, L.; Li, H.; Zhou, H.; Zhao, X.; Zhan, X.; Tan, J. Multiple sources of the Early Mesozoic Gouli batholith, Eastern Kunlun Orogenic Belt, northern Tibetan Plateau: Linking continental crustal growth with oceanic subduction. Lithos 2017, 292-293, 161-178. [CrossRef]

67. Wang, Q.; Wyman, D.A.; Xu, J.F.; Zhao, Z.H.; Jian, P.; Xiong, X.L.; Bao, Z.W.; Li, C.F.; Bai, Z.H. Petrogenesis of Cretaceous adakitic and shoshonitic igneous rocks in the Luzong area, Anhui Province (eastern China): Implications for geodynamics and $\mathrm{Cu}-\mathrm{Au}$ mineralization. Lithos 2006, 89, 424-446. [CrossRef]

68. Huang, X.L.; Xu, Y.G.; Lan, J.B.; Yang, Q.J.; Luo, Z.Y. Neoproterozoic adakitic rocks from Mopanshan in the western Yangtze Craton: Partial melts of a thickened lower cnust. Lithos 2009, 1, 367-381. [CrossRef]

69. Zhu, D.C.; Li, S.M.; Cawood, P.A.; Wang, Q.; Zhao, Z.D.; Liu, S.A.; Wang, L.Q. Assembly of the Lhasa and QIangtang terranes in central Tibet by divergent double subduction. Lithos 2016, 245, 7-17. [CrossRef]

70. Zhu, D.C.; Pan, G.T.; Wang, L.Q.; Mo, X.X.; Zhao, Z.D.; Zhou, C.Y.; Liao, Z.L.; Dong, G.C.; Yuan, S.H. Tempo-spatial variations of Mesozoic magmatic rocks in the Gangdise belt, Tibet, China, with a discussion of geodynamic setting-related issues. Geol. Bull. China 2008, 27, 1535-1550.

71. Pearce, J.A.; Harris, N.B.W.; Tindle, A.G. Trace-element discrimination diagrams for the tectonic interpretation of granitic-rocks. J. Petrol. 1984, 25, 956-983. [CrossRef] 